June, 1996

MSUHEP-60426

CTEQ-604

\title{
Improved Parton Distributions from Global Analysis of Recent Deep Inelastic Scattering and Inclusive Jet Data †
}

\author{
H. L. Lai ${ }^{c}$, J. Huston ${ }^{c}$, S. Kuhlmann ${ }^{a}$, F. Olness ${ }^{e}$, J. Owens ${ }^{b}$, D. Soper ${ }^{d}$, \\ W. K. Tung ${ }^{c}$, H. Weerts ${ }^{c}$ \\ ${ }^{a}$ Argonne National Laboratory, ${ }^{b}$ Florida State University, ${ }^{c}$ Michigan State University, \\ ${ }^{d}$ University of Oregon, ${ }^{e}$ Southern Methodist University
}

\begin{abstract}
The impact of recent precision measurements of DIS structure functions and inclusive jet production at the Tevatron on the global QCD analysis of parton distribution functions is studied in detail. Particular emphasis is placed on exploring the range of variation of the gluon distribution $G(x, Q)$ allowed by these new data. The strong coupling of $G(x, Q)$ with $\alpha_{s}$ is fully taken into account. A new generation of CTEQ parton distributions, CTEQ4, is presented. It consists of the three standard sets $(\overline{\mathrm{MS}}$, DIS and leading order), a series that gives a range of parton distributions with corresponding $\alpha_{s}$ 's, and a set with a low starting value of $Q$. Previously obtained gluon distributions that are consistent with the high $E_{t}$ jet cross-section are also discussed in the context of this new global analysis.
\end{abstract}

\footnotetext{
$\dagger$ This work was partially supported by DOE and NSF
} 


\section{Introduction}

Lepton-lepton, lepton-hadron, and hadron-hadron interactions probe different and complementary aspects of Quantum Chromodynamics. Each of these interactions provides a window onto the elementary interactions of quarks and gluons and probes the running coupling and quark masses. The corresponding calculations are performed using perturbative Quantum Chromodynamics (pQCD). In addition these interactions probe the partonic structure of hadrons, as represented by the parton distribution and fragmentation functions [1] [2]. These functions are essentially non-perturbative. There are, of course, large areas of overlap between various processes, which provide impressive consistency checks of the theory. In the first approximation, lepton-lepton processes provide clean measurements of basic parameters such as quark charges, the strong coupling $\alpha_{s}(Q)$, and fragmentation functions of partons into hadrons. Deep inelastic scattering structure functions and lepton-pair production cross-sections in hadron collisions provide the main source of information on the quark distributions $f^{q}(x, Q)$ inside hadrons. At leading order, the gluon distribution function $G(x, Q)$ enters directly in hadron-hadron scattering processes with direct photon and jet final states. In a global QCD analysis incorporating all these processes, one tries to exploit the strengths of each process in a uniform framework. Modern analyses are carried out to at least next-to-leading order (NLO), thus $\left\{\alpha_{s}(Q), f^{q}(x, Q), G(x, Q)\right\}$ all contribute and mix in the theoretical formulas for each process. However, the broad picture outlined above does reflect the main roles the various processes play in the analysis.

Direct photon production has long been regarded as potentially the most useful source of information on $G(x, Q)$. Fixed-target direct photon data, especially those from WA70 [3], have been widely used in existing global analyses. However, there are a number of theoretical uncertainties which affect the predictions of the normalization and slope of the measured direct photon $p_{t}$ spectrum. These effects include: (1) the sensitivity of the theoretical calculations to the choice of factorization and renormalization scales [四; (2) $k_{t}$ broadening of the initial state partons due to soft gluon radiation [4]; and (3) photon fragmentation uncertainties [4] and the related issue of photon isolation cuts [5]. When all these uncertainties are taken into account, existing direct photon data do not place as tight constraints on the gluon distribution as is commonly believed [6]. Full exploitation of the potential of this process in a QCD global analysis will require significant progress in the understanding of the above issues.

An important process that is sensitive to gluons is jet production in hadron-hadron collisions. In leading order, the cross-section is proportional to $\alpha_{s}^{2}(Q) G(x, Q) G\left(x^{\prime}, Q\right)$ and $\alpha_{s}^{2}(Q) G(x, Q) q\left(x^{\prime}, Q\right)$ for the gluon-gluon and gluon-quark scattering subprocesses respectively. Experimental measurement of various inclusive jet cross-sections has progressed to an increasingly quantitative level in recent years. For instance, at the Tevatron, good data 
on single jet production are now available over a wide range of transverse energy, $15 \mathrm{GeV}$ $<E_{t}<450 \mathrm{GeV}$ [7, 8]. NLO QCD calculations of jet cross-sections have also reached a mature stage [9, 10, 11]. Many issues relating to jet definition (which is important for comparing theory with experiment) encountered in earlier stages of jet analysis have been extensively studied and are better understood. For the moderate to large $E_{t}$ range, the scale dependence of the NLO inclusive jet cross section turns out to be relatively small [12]. Thus, it is natural that inclusive jet data should now be incorporated into a global QCD analysis, and that these data should play a role in constraining the gluon distribution $G(x, Q)$.

We have carried out a first systematic study of this problem using the CTEQ global analysis framework 13. I In this paper, we shall focus on the question: How well can the gluon distribution be determined as the result of recent advances in experimental measurements? We discuss phenomenological issues pertinent to extracting $G(x, Q)$ in the global analyses. These factors are systematically taken into account in a series of analyses to gain insight on the current range of uncertainties on $G(x, Q)$. We found that recent, more precise, DIS data have a significant influence in narrowing down the parton distribution functions (PDF's), including $G(x, Q)$; and the inclusion of inclusive jet data from hadron colliders further solidifies knowledge on $G(x, Q)$ over a wide range of $x$. As the result of this study, we present new sets of CTEQ parton distributions (in $\overline{M S}$, DIS and LO schemes) as well as a series of distributions which give a range of variation of PDF's consistent with current data. We give quantitative information on how these distributions compare to the data sets used in the analysis. In addition, we provide a new set of PDF's with a low initial $Q_{0}^{2}=0.5 \mathrm{GeV}^{2}$; and we discuss the previously obtained gluon distributions designed to accommodate the high $E_{t}$ jets [6] in the context of the CTEQ4 analysis.

\section{Issues on the determination of the gluon distribution}

In pQCD, the gluon distribution function is always accompanied by a factor of the strong coupling (i.e. it appears as $\alpha_{s} G(x, Q)$ ), both in the hard cross-sections and in the evolution equation for the parton distributions. Thus, the determination of $\alpha_{s}$ and $G(x, Q)$ is in general a strongly coupled problem. In principle, $\alpha_{s}$ can be independently extracted from $e^{+} e^{-}$collisions, or in sum rule measurements in deep inelastic scattering. $G(x, Q)$ can then be determined in a global analysis, along with the quark distributions $f^{i}(x, Q)$, by treating $\alpha_{s}$ as known. Alternatively, one can try to determine $\alpha_{s}, G(x, Q)$ and the quark distributions at once in a global analysis. This relies on the full $(x, Q)$ dependence of the wide range of data to differentiate $\alpha_{s}$ (which controls the overall $Q$-dependence of all quantities) from the parton distributions (which depend on both $x$ and $Q$ ). This method is not as "clean" as the

\footnotetext{
${ }^{1} \mathrm{~A}$ similar analysis was carried out earlier [6], focusing on the interpretation of the "high $p_{t}$ excess" seen in the CDF jet measurement [7]. See Sec. 6 for a discussion of the relation of [6] to the current analysis.
} 
first approach, and it will not become precise until the global analysis system has become better constrained. Eventually, however, it is important to demonstrate that the same value of $\alpha_{s}$ consistently describes all the processes included in the global analysis. Hence, the two approaches are indeed complementary.

It is well known that, at present, the value of $\alpha_{s}$ determined at high energy colliders, especially LEP, is generally higher than that obtained from analyses of fixed-target DIS data [14. Since global QCD analyses are up to now dominated by the copious high statistics DIS data, they favor values of $\alpha_{s}$ close to the lower "DIS value". This situation may change when more and more quantitative results from hadron collider processes, such as inclusive jet and direct photon production, are included in the global analysis. In the following, we shall explore the range of variation of $G(x, Q)$ when the value of $\alpha_{s}$ is varied within the currently accepted region, which we shall take to be $0.105<\alpha_{s}\left(M_{Z}\right)<0.122$. 15. The problem of the determination of $\alpha_{s}$ in global analysis and the question about consistency of $\alpha_{s}$ among different processes will be considered in a subsequent study [16].

For a quantitative study of $G(x, Q)$, another relevant consideration is: How does the choice of parametrization of the initial gluon distribution at some $Q=Q_{0}$ affect the results? All global analyses use a generic form:

$$
G\left(x, Q_{0}\right)=A_{0} x^{A_{1}}(1-x)^{A_{2}} P\left(x ; A_{3}, . .\right)
$$

with $A_{1,2}$ being physically associated with small- $x$ Regge behavior and large- $x$ valence counting rules respectively; and $P\left(x ; A_{3}, \ldots\right)$ being a suitably chosen smooth function depending on one or more parameters. In general, both the number of free parameters and the functional form can have an influence on the global fit. In the CTEQ3 analysis [13], an effort was made to minimize the number of free parameters, resulting in an economical set whereby $A_{1}^{G}=A_{1}^{\text {sea }}$, and $P_{C T E Q 3}\left(x ; A_{3}\right)=1+A_{3} x$. We shall refer to this choice as the minimal set in the following discussions. In the literature, more degrees of freedom have been assigned to $G\left(x, Q_{0}\right)$. For instance, in CTEQ2 [13] and in recent MRS fits [17], $A_{1}^{G}$ is allowed to vary independently of $A_{1}^{\text {sea }}$; and the function $P$ contains one more free parameter than $P_{C T E Q 3}$ : $P_{C T E Q 2}\left(x ; A_{3}, A_{4}\right)=1+A_{3} x^{A_{4}} ; P_{M R S}\left(x ; A_{3}, A_{4}\right)=1+A_{3} \sqrt{x}+A_{4} x$. Since two extra degrees of freedom are added, we shall refer to this class of parametrization as $(m+2)$ - i.e. minimal plus two. The more general parametrization clearly allows a wider range of variation of $G\left(x, Q_{0}\right)$. Some pertinent questions are: whether these general parametrizations are required by current data; and do these parametrizations give a good indication of the range of variation of $G\left(x, Q_{0}\right)$ ? We shall investigate these questions in some detail in the next two sections.

Finally, although the PDF's determined from global analysis should, in principle, be universal, they could, in practice, depend on the choice of data sets - in particular, on the choice of " $Q_{c u t}$ " values that specify the minimum hard physical scale $\left(Q, P_{t}, \ldots\right)$ required for 
data points in the various physical processes to be included in the fit. If the NLO QCD theory is truly applicable in the kinematic range of the data, the parton distributions should be insensitive to the value of $Q_{c u t}$. Since current theory does not predict what value $Q_{c u t}$ should take for each process, this point has to be investigated phenomenologically.

\section{Impact of recent DIS data on the global analysis of parton distributions}

Since the publication of the CTEQ3 analysis, more accurate and extensive DIS data from NMC [18] and HERA [19, 20] as well as new data from E665 [21] have become available. In comparing the new data with NLO QCD $F_{2}$ computed from CTEQ3M distributions, we find general agreement, except for the small- $x$ region where the more precise recent data show deviations from the theory curves. This is shown in Fig. 1 for the NMC and H1 data sets respectively.[2 Thus, we first update the CTEQ3 analysis under several different conditions, in order to study the impact of these new DIS data on the global analysis of parton distributions, especially the extraction of the gluon distribution.

The magnitude of the uncertainty in $G\left(x, Q_{0}\right)$ due to the current uncertainty on $\alpha_{s}$, will be investigated by systematically varying the value of $\alpha_{s}$ over the interval $0.105<\alpha_{s}<0.122$, as mentioned in the previous section. We shall use the short-hand $\alpha_{s}$ for $\alpha_{s}\left(M_{Z}\right)$ throughout. In terms of $Q C D$ Lambda values, this range of $\alpha_{s}$ corresponds to $100<\Lambda_{5}^{\overline{M S}}<280(\mathrm{MeV})$ and $155<\Lambda_{4}^{\overline{M S}}<395(\mathrm{MeV})$. We shall in general use the $\overline{M S}$ scheme in NLO QCD.

To provide a base-line for comparison, we first obtain a series of such fits under identical conditions and using the same data sets (i.e. pre-1995) as in the CTEQ3 analysis 13. We shall refer to this as the A-series.'p By definition, the best fit in this series is the published CTEQ3M fit with $\alpha_{s}=0.112\left(\Lambda_{5}^{\overline{M S}}=158 \mathrm{MeV}\right)$. A comparison of the gluon distributions that correspond to these values of $\alpha_{s}$ are presented in Fig. 3. In order to render the differences in the various regions of $x$ visible over the range $10^{-4}<x<1$, part (a) highlights the small$x$ region by plotting $x G(x, Q)$ against $\log x$, part (b) accentuates the medium- $x$ range by plotting $x^{2} G(x, Q)$ vs. $\log x$. For the many detailed comparisons to follow, these separate plots, though conventional, will prove to be rather cumbersome. We consolidate them into one single less-conventional plot

\footnotetext{
${ }^{2}$ Results are similar for E665 and ZEUS. Comparison with the full data sets will be presented later, cf. Fig. 2

3 This series of fits were originally obtained in 1994. They have been used in various phenomenological studies related to gluon distributions and $\alpha_{s}$ determination conducted by CTEQ, CDF, and D0 Collaborations. They have not been formally published.

${ }^{4}$ Note that, since $x^{2} G(x) \cdot d \log x=x G(x) \cdot d x=$ momentum fraction carried within $d x$, each curve in this plot directly depicts the distribution of the momentum fraction carried by the gluon for that set.
} 
in Fig. $\$$ in which all curves are normalized by the function $x^{-1.5}(1-x)^{3}$, which takes out most of the singular (rapidly vanishing) factors at small (large) $x$. The scale for the abscissa is chosen to be a function of $x$ which smoothly interpolates between $\log x$ (at small $x$ ) and $x$ (at large $x$ ) so that the behavior of $G(x, Q)$ over the full $x$ range is more evenly displayed. We see that all of the features seen in the three plots of Fig. 3 are evident in this single figure. This will be the format of choice in most subsequent comparisons.

We see in Fig. 1 that, in the region $x>0.05$ where the largest concentration of data used for the fit lie, increasing values of $\alpha_{s}$ lead to decreasing values of $G(x, Q)$ - as expected (particularly for the direct photon data) since the product of the two enters into most cross-section and evolution kernel formulas. f As noted before, in the CTEQ3 analysis, and therefore in this series of fits, the initial gluon distribution function is parametrized minimally as

$$
G\left(x, Q_{0}\right)=A_{0} x^{A_{1}}(1-x)^{A_{2}}\left(1+A_{3} x\right)
$$

with $A_{1}$ set to be the same as that of the sea quarks. Hence there are 3 free gluon parameters - $A_{0,2,3}$ - in the fit. For each $\alpha_{s}$, we found the best solution to be quite stable against perturbations in the fitting procedure and starting parameters, indicating the parametrization and the experimental constraints are well-matched. This also results in an orderly variation of $G(x, Q)$ as $\alpha_{s}$ is varied, as seen in the figure. If one takes the range of $\alpha_{s}$ used here as representing the current uncertainty on $\alpha_{s}$, then the spread of the gluon distribution shown in Fig. 4 gives the corresponding uncertainty on $G(x, Q)$ (based on the data available prior to 1995 , and on the variation of $\alpha_{s}$ alone). We should mention that, although quark distributions are allowed to vary freely, the valence quark distributions remain practically the same for all of the fits in this series, as they are very much pinned down by the precision DIS data in the region where they dominate the structure functions. On the other hand, the sea quark distributions couple to $G(x, Q)$; thus they do show a systematic variation with $\alpha_{s}$, although the variation is somewhat reduced compared to that of the gluon.

Next, we investigate the impact of the new DIS data from NMC [18], E665 [21] and HERA [19, 20 on $F_{2}$ by repeating the same study, with the new data sets replacing the original ones. The resulting series of fits is called the B-series. The quality of these fits (measured in $\chi^{2}$ values) are similar to those of the A-series. Six representative gluon distributions in this series are shown in Fig. 5 along with that of CTEQ3M for reference. It is rather striking to note that the spread in $G(x, Q)$ observed above in the small- $x(<0.01)$ region has been practically eliminated. This is precisely the region covered by the HERA experiments. In addition, the new gluons are shifted down from those of the A-series in the region $0.05<x<0.3$ where all three DIS experiments contribute. At first glance, this may appear surprising in view of the conventional wisdom that $F_{2}$ data are only sensitive to quarks, not gluons.

\footnotetext{
${ }^{5}$ The order is reversed for small $x$, because of the momentum sum rule.
} 
However, we must realize that, first, in the small- $x$ region $G(x, Q)$ is quite large-typically about 20 times bigger than the quark distributions - thus it has a strong influence, directly and indirectly, on all physical quantities through the hard cross-section and the evolution equation. Moreover, these fits use the minimal parametrization, including the constraint $A_{1}^{G}=A_{1}^{\text {sea }}$ which strongly couples the behavior of $G(x, Q)$ at small- $x$ to that of sea quarks. Thus, the much better determined $G(x, Q)$ just reflects the improved accuracy of new data in this region. We note also, the large- $x$ behavior of the new series is somewhat different from the A-series, even if there are no new data in that region. This must be due to the indirect effect of the required changes below $x=0.1$, induced by the restrictive functional form Eq. 2, and the constraint imposed by the momentum sum rule. We should point out that the absolute value of the gluon distribution in the region above $x=0.5$ is very small (about $10^{-3}$ compared to its value at $x=0.1$ ); thus the significance of the observed differences should not be over-emphasized.

The minimal parametrization for $G\left(x, Q_{0}\right)$ used above was originally chosen in the CTEQ3 analysis for its economy - all data sets included in these global analyses can be reasonably well fitted with this form. This does not prove that the true gluon distribution must fall within the range shown above; in particular, the true $G\left(x, Q_{0}\right)$ may be more complicated than can be represented by this parametrization. (For instance, all global analyses find it necessary to use one more parameter to describe the valence quarks.) Only experiments probing $G(x, Q)$ in a different way can tell whether our results so far are adequate. Before turning to such additional input, we can obtain a different estimate of the uncertainty on the gluon distribution that is complementary to the width of the "band" shown in Figs. 世5. We adopt the more general " $(\mathrm{m}+2)$ " parametrization of $G\left(x, Q_{0}\right)$ already used in CTEQ2:

$$
G\left(x, Q_{0}\right)=A_{0} x^{A_{1}}(1-x)^{A_{2}}\left(1+A_{3} x^{A_{4}}\right)
$$

In addition to introducing the new parameter $A_{4}$ compared to Eq. 2, the parameter $A_{1}$ is untied from $A_{1}^{\text {sea }}$ and treated as free. This results in a new series of fits, called the C-series.

With two more free parameters than in the B-series, one would expect (i) to fit the collective data "better" than before and (ii) to find an increased range of variation of the gluon distribution. Indeed, the $\chi^{2}$ for the fits decreased slightly (by about 10 (/1000 pts.)) compared to the corresponding ones in the B-series. The gluon distributions at $Q=5 \mathrm{GeV}$ in this series for 6 values of $\alpha_{s}$ is shown in Fig. 6. First, we see that the range of variation of $G(x, Q)$ in this series is much wider as compared to that of series B, although both include the same improved DIS data. In particular, in the small- $x$ region the very narrow range in series $\mathrm{B}$ is very much opened up by the freeing of the $A_{1}$ parameter for the gluon - since now $q(x, Q)$ and $\alpha_{s} G(x, Q)$ can vary independently, the measured $F_{2}$ (which depends on both) no longer constrains each piece tightly as in the B-series. Secondly, we note that the gluon distribution does not vary in a systematic manner as the $\alpha_{s}$ value is varied - in contrast 
to the well-constrained case in series A and B. Further study has indicated that, unlike in the other cases, small changes in the fitting process can lead to different solutions for some values of $\alpha_{s}$. This suggests that the fits are not entirely stable; or, in other words, the system becomes somewhat under-constrained with the two extra parameters introduced.

These observations point to the need for more experimental input in order to better measure the gluon distribution. We need new data to determine whether the additional degrees of freedom associated with $A_{1}^{G}$ and $A_{4}^{G}$ are required for the true gluon or whether the restricted form used in series $\mathrm{B}$ is already sufficient. If $A_{1}^{G}$ and $A_{4}^{G}$ are required, these new data could help to stabilize the fits found in the $\mathrm{C}$-series and hence shed light on the possible range of $G(x, Q)$ allowed. From the discussion given in the introduction, it is clear that inclusive jet production data could be used to help resolve these issues, as we will show in the next section. To conclude this section, Table [ 1 summarizes the above described three series of global fits, as well as those including jet data to be discussed next.

\section{Comparison with New Inclusive Jets Cross-section}

For studying the impact of inclusive jet production cross-section, we use the recent measurement of $d \sigma / d E_{t}$ from the CDF [7] and D0 [8] Collaborations. The preliminary data obtained in run IB of the Tevatron by the two experiments are shown in Fig. 7. Although data are available for $15 \mathrm{GeV}<E_{t}<450 \mathrm{GeV}$, we will include in our NLO QCD analysis only data above $50 \mathrm{GeV}$ because there are a number of potential theoretical and experimental problems that may affect the proper comparison between NLO QCD theory and data for lower $E_{t}$. These include (1) scale uncertainty of NLO QCD calculations, which becomes non-negligible at low $E_{t}$ (cf. Fig. 8a); (2) ambiguities in the definition of the "underlying event" coming from the proton-antiproton remnants (cf. Fig. \&b); (3) possible problems in the match between theoretical and experimental jet definitions, such as fragmentation products outside the jet cone; (4) $k_{t}$ broadening of the initial state partons [4]; and (5) non-perturbative corrections to the theory, which could be of order $1 / E_{t}$ rather than $1 / E_{t}^{2}$ [22]. All of these affect low $E_{t}$ jets much more than high $E_{t}$ jets, as will be illustrated by two examples, one theoretical and one experimental. Fig. 8a shows the scale-dependence of the NLO QCD calculation as a function of $E_{t}$ : the theoretical inclusive jet cross-section is shown for several choices of the renormalization and factorization scale $\left(\mu=\mu_{R}=\mu_{F}\right)$ normalized to our standard choice $\mu=E_{t} / 2$. For low $E_{t}$, the ratio becomes large and unstable; above $50-75 \mathrm{GeV}$, the different choices are within $10 \%$ and stay relatively constant - they amount to shifts in the overall normalization of the cross-section. Fig. $8 \mathrm{~b}$ shows the percentage effect on the inclusive jet cross-section in the CDF experiment due to a $\pm 30 \%$ change in the underlying

\footnotetext{
${ }^{6}$ The theoretical calculations of jet cross-section in this paper are carried out using the EKS program [9].
} 
event correction (in Run IA). Again, the uncertainty becomes large below $50-75 \mathrm{GeV}$.

To emphasize the quantitative aspects of the subsequent analysis, the measured steeply falling $d \sigma / d E_{t}$ is normalized to the NLO QCD theoretical expectation using the CTEQ3M parton distributions (solid horizontal line) and displayed in Fig. 9 on a linear plot (with statistical errors only on the data points). In Fig. 9, we have taken into account the slightly different pseudo-rapidity coverage of the two experiments $(0.1<|\eta|<0.7$ for CDF vs. $|\eta|<0.5$ for D0) by normalizing each data set with respect to the theory values computed with the corresponding $\eta$ range. In addition, we have allowed a small overall normalization of the two data sets, well-within the quoted uncertainties, for this comparison. This figure shows that the two data sets agree quite well over the entire $E_{t}$ range, especially when considering the quoted systematic uncertainties (not shown). See Ref. 23 for more discussions. We will discuss the experimental systematic uncertainties in the proper context of the "range" of gluon distributions later in this paper. The rise of the data points at high $E_{t}$ values over the CTEQ3M expectation, more noticeable for the CDF points, has been the subject of much recent discussion and speculation [7, 6, 24]. We will comment on this issue in the context of the global analysis conducted in this paper in a later section.

Since most inclusive jet data are collected in the central rapidity region, the $x$-value of the PDF's probed is around $x_{t}=2 E_{t} / \sqrt{s}$. For $50 \mathrm{GeV}<E_{t}<450 \mathrm{GeV}$, the $x$ range is approximately $0.06-0.5$. Over this range, the relative importance of the three parton subprocesses - quark-quark, quark-gluon, and gluon-gluon - shifts continuously from being gluon-dominated to quark-dominated, as illustrated in Fig. 10. We should also keep in mind that these jet data probe hadron structure at much higher momentum scales than fixed-target experiments. Due to the nature of the QCD evolution equation, parton distributions at these high momentum scales are determined by those at lower scales and higher $x$ values. Thus the effective $x$-range in $G\left(x, Q_{0}\right)$ for some $Q_{0}$, say $1.6 \mathrm{GeV}$ used in our analysis, probed by these jet data extends to much higher values than the nominal values mentioned above. Since the quark distributions throughout this range are very well pinned down by DIS experiments, one expects the jet data to be particularly useful in constraining the gluon distribution. The value of $\alpha_{s}$ has considerable influence on the gluon determination for several reasons. First, the cross-section for medium $x_{t}$ is proportional to $\alpha_{s}^{2} G^{n}(x, Q)(\mathrm{n}=2,1,0)$, so that as $\alpha_{s}$ increases, $G(x, Q)$ will decrease. Second, $\alpha_{s}$ controls the rate of evolution of $G(x, Q)$ and hence affects the slope of the gluon distribution for given measured jet cross-sections. Third, $\alpha_{s}(\mu)$ itself depends on $x$ through $\mu=E_{t} / 2=x \sqrt{s} / 4$ (at $\eta=0$ ), so that the rate of variation of $\alpha_{s}$ (controlled by its strength) is coupled to the $x$-dependence of $G(x, Q)$ in the cross-section formula.

We now apply the results obtained in Sec. 3 to these jet data to see how the latter agree with the predictions of perturbative QCD using these new parton distributions determined by the other processes. Fig. 11] compares the predictions of the PDF's from the B-series 
(which incorporate the most recent DIS data and use the minimal parameters for the gluon) with the jet data, using the same "(Data - Theory) / Theory" format as Fig. 9. We use the set with $\alpha_{s}\left(M_{Z}\right)=0.116$ as the "Theory" (horizontal solid line) against which the data points as well as the predictions of the other fits with different $\alpha_{s}$ values in the series are displayed in this plot. To make these comparisons, we allow an overall relative normalization between theory and data. The normalization factor for the CDF/D0 data set ranges from 0.94/0.92 to $1.08 / 1.06$ for $\alpha_{s}=0.110$ to 0.122 . [25] The normalization uncertainties quoted by the CDF and D0 experiments are around 5\%. Considering the 7 orders of magnitude of variation of the cross-section (Fig. 7), this is quite remarkable. Within the minimal parametrization of the gluon used by the B-series, the parton distributions narrowed down by recent precise DIS data (see previous section) are remarkably consistent with the new high statistics inclusive hadron-hadron jet data. We also found that the more generally parametrized C-series PDF's give qualitatively similar predictions for jet cross-sections compared to the B-series displayed in Fig. 11; hence they will not be separately shown.

The important questions at this point are the following: (i) At a more quantitative level, how can these parton distributions be improved by including the jet data in the analysis from the beginning; and (ii) will the addition of the jet data reduce the variation of $G(x, Q)$ when we use the more general $(\mathrm{m}+2)$ parametrization?

\section{New CTEQ parton distribution sets - CTEQ4}

To answer these questions, we have performed an extensive study of the interplay of the inclusive jet data with the high-precision DIS and other data within the CTEQ QCD global analysis program. The complete set of processes and experiments used is given in Table 2. To display explicitly the wide coverage of these experiments over the kinematical variables, we show in Fig. 121 a map of the $(x, Q)$ plane with the data range of the various experiments. We see the greatly expanded kinematic coverage compared to a few years ago: in the direction of small- $x$ due to the HERA experiments, and in the high $Q$ direction due to the Tevatron inclusive jet experiments. 5 As before, all processes are treated consistently to NLO accuracy in PQCD. This new round of global analysis will be referred to as the CTEQ4 analysis.

Building upon studies described in the previous sections, we explored all the issues described in Sec. 2, now with jet data also playing a role. Although the quark distributions are coupled to $G(x, Q)$ and $\alpha_{s}$, they remain tightly constrained by the DIS experiments, hence stay very close to those determined before. Thus, our studies concern again mainly

\footnotetext{
${ }^{7}$ Such a renormalization, within errors, is usually allowed in global fitting.

${ }^{8}$ Since these experiments are the only ones in the respective kinematic region, new information on parton distributions extracted from these data provide challenges to QCD theory for future comparison with independent measurements based on other processes.
} 
the range of variation of $G(x, Q)$ due to uncertainties in $\alpha_{s}$ and the parametrization of the non-perturbative initial distribution. (We have also looked into the influence due to the choice of " $Q_{\text {cut }}$ ", which will be described in the Appendix). Since the results from Sec. 1 indicate that it is possible to obtain good fits to all the data using the minimal parametrization of the gluon distribution even without taking into account the experimental systematic errors on the inclusive jet data, we anticipate the most important role of the latter in the new analysis is to constrain the possible range of $G(x, Q)$. Hence, we shall use the more general $(\mathrm{m}+2)$ parametrization which allows a wider range of variation of $G(x, Q)$. We shall not include the correlated systematic uncertainties on the jet data since they are not crucial for the present purposes. This point will come up again later. More discussions on the experimental systematic uncertainties can be found in the Appendix.

The new generation of CTEQ4 parton distributions are summarized in Table 3. They will be described in turn in the following.

\section{Standard CTEQ4M parton distributions}

We first present the standard fit in the $\overline{M S}$ scheme which we will designate as the CTEQ4M set of parton distributions. The $\alpha_{s}\left(m_{Z}\right)$ value for this set is 0.116 , corresponding to second order $\Lambda_{5}=0.202$ or $\Lambda_{4}=0.296 \mathrm{GeV}$. This set gives excellent fit to all data sets. The total $\chi^{2}$ for 1297 DIS and DY data points is 1320. Detailed information on the $\chi^{2}$ 's for the various experiments, in comparison to those obtained using other current and previous generations of parton distributions are presented in Tables 4 and 5 respectively. The direct photon and jet data sets are not included in the $\chi^{2}$ table since, without including the sizable theoretical uncertainties for the formerf and experimental systematic errors for the latter, the significance of such $\chi^{2}$ values would be difficult to evaluate. The comparison of the CDF and D0 jet data to the NLO QCD inclusive jet cross-section calculated with the CTEQ4M distributions is shown in Fig. 13. And the comparison of the recent NMC, H1, and ZEUS data sets to the fit is shown in Figs. 2.

From Table 4, we see that the CTEQ4M PDF set has the best overall quantitative agreement between NLO QCD theory and global data on high energy scattering. It also represents a significant improvement over the previous generation of parton distributions, as a comparison to Table 5 makes clear. Most of the difference is caused by the new precision data from the HERA experiments. Fig. 13 shows good general agreement of CTEQ4M with the jet data, while the much discussed "high $E_{t}$ excess" is still noticeable. We will return to this issue in Sec. 6 where an alternative "high $E_{t}$ jet-fit" CTEQ4HJ (included in Table 甘) will be discussed. Figs. 2 explicitly shows the improvement of CTEQ4M over CTEQ3M in describing the recent high-precision DIS experiments. In the Appendix, we will give detailed

\footnotetext{
${ }^{9}$ See the Introduction and Refs. [4, 6] for discussions on these uncertainties.
} 
information on the parameters which characterize the initial parton distributions at $Q_{0}=1.6$ $\mathrm{GeV}$ (which coincides with our choice of the charm threshold). Here, we only note that the $\left(A_{1}, A_{2}\right)$ parameters (cf. Eqs. [ \& 2) of the gluon and the sea quarks are $(-1.21,4.67)$ and $(-1.14,8.04)$ respectively.

\section{CTEQ4A-series of parton distributions with varying $\alpha_{s}$ and $G(x, Q)$}

In exploring the range of variation of allowed $G(x, Q)$ by varying the values of $\alpha_{s}$, changing the number of parameters for the gluon, and altering the $Q_{c u t}$ of data selection, we have found the largest effect is due to the varying of $\alpha_{s}$. Hence, in presenting a series of PDF's which give a reasonable representation of the range of possibilities, we use those generated with an $\alpha_{s}$ range centered around the CTEQ4M value of 0.116 , which is close to the current world average [14]. This series will be designated as CTEQ4A-series (shorthand for CTEQ4Alpha) - CTEQ4A1, ...,CTEQ4A5, with CTEQ4A3 being the same as CTEQ4M. The $\chi^{2}$ per point for the 1297 non-jet data points are $(1.07,1.02,1.02,1.07,1.19)$ respectively. The higher $\chi^{2}$ values at low values of $\alpha_{s}$ mainly come from the HERA DIS experiments; the higher $\chi^{2}$ values at high values of $\alpha_{s}$ are mainly due to the fixed-target DIS experiments [25]. The difference in $\chi^{2}$ above minimum, especially for the highest value of $\alpha_{s}$, is larger than in previous CTEQ analyses (e.g. CTEQ2ML vs. CTEQ2M) due to the sharply reduced errors on recent DIS data. However, the difference is comparable to that between the MRSJ and CTEQ4M $\chi^{2} s$, cf, Table G. Because correlations in the experimental errors are not available for all experiments, hence have not been included in current global analyses, and since theoretical uncertainties are even harder to quantify, pragmatically, we take these $\chi^{2}$ differences as being acceptable for present purposes.

Fig. 14 shows the comparison of the CTEQ4A parton distribution sets with the two jet data sets, using CTEQ4M as the common calibration. The overall normalization factor on the jet data sets applied to the various fits range from 0.96 (for CTEQ4A1 on D0 points) to 1.02 (for CTEQ4A5 on CDF points), well within the experimental uncertainty of $\sim 5 \%$. Comparing to Fig. 11 and the range of normalization factors needed there $(0.92-1.08$, which is wider than the experimental error), we see the expected improvement of the agreement with the jet data.

The gluon distributions associated with the various values of $\alpha_{s}$ in this series are shown in Fig. 15. Comparing the CTEQ4A-series to the C-series (same parametrization form for $G\left(x, Q_{0}\right)$ ), we see that the constraining influence of the jet data has a rather dramatic effect. The unstable behavior of the various curves observed in the C-series has been replaced by an orderly variation as one steps through the values of $\alpha_{s}$ within the range explored. We found, indeed, that for each value of $\alpha_{s}$, the solution of $G(x, Q)$ is rather unique against perturbations in the fitting procedure. 
One concern is that the variation in the CTEQ4A series is too small due to the lack of treatment of systematic uncertainties in the jet data. To address this issue, we compare the change in the calculated jet cross-sections between the extremes of the CTEQ4A series to the largest $E_{t}$-dependent uncertainty in the CDF data. See Fig. 16. It shows that the range of variation in the CTEQ $4 \mathrm{~A}$ series is about $10 \%$ in the moderate $E_{T}$ range, while the experimental systematic uncertainty is about the same. This observation lends some confidence that this series gives a reasonable estimate of the range of variation of $G(x, Q)$. To the extent that there are sources of uncertainty other than $\alpha_{s}$, the variation in $G(x, Q)$ given here may be considered a minimum range. However, our study does indicate that the variation due to the uncertainty of $\alpha_{s}$ may be the dominant one.

Fig. 17 shows a comparison of some of the new gluon and singlet quark distributions with those of CTEQ3M and MRSJ in the usual form $x f(x, Q)$ without the normalization factor as in previous figures. On this conventional plot, differences in $G(x, Q)$ can be seen only in the small- $x$ region, and the CTEQ3M and CTEQ4M gluons appear to be indistinguishable. Differences in the singlet quark distribution are more evident near $x=0.01$. The fact that only small changes in the parton distributions result from adding so much new data in the global analysis is testament to the impressive progress in pinning down these parton distributions that has been made in recent years. These changes, though small, are nonetheless physically significant, as demonstrated by the substantial differences in $\chi^{2}$ values between the new and old parton distribution sets on the precision experiments given in Tables 1 and 5 .

\section{Other CTEQ4 parton distributions}

Along with the standard CTEQ4M $\overline{M S}$ parton distributions, we have also obtained corresponding parton distributions in the "DIS scheme" - CTEQ4D. CTEQ4D uses the same value of $\alpha_{s}(=0.116)$ as CTEQ4M; it is obtained by fitting under identical conditions as CTEQ4M except that the hard cross-sections are evaluated in the DIS scheme. The $\chi^{2}$ values of this fit are comparable to those of CTEQ4M. In addition to these two standard sets, for applications requiring leading order ( $\mathrm{LO}$ ) calculations and low values of the scale $Q$ (LQ), we also provide appropriate parton distribution sets labelled CTEQ4L and CTEQ4LQ respectively. The CTEQ4LQ set can be used for $Q^{2}>Q_{i}^{2}=0.5 \mathrm{GeV}^{2}$. It was obtained by fitting the same data sets as the other PDF sets. Since the proper treatment of low $Q$ data must involve more physics input (such as higher twist effects) than included here, CTEQ4LQ

\footnotetext{
${ }^{10}$ Of course, given the good agreement between the two Tevatron experiments [23], if the CDF jet data requires a significant change due to a systematic error, the D0 data would require the same change, an unlikely occurrence since there is almost no correlation in the two experimental measurements.

${ }^{11}$ Below this scale $(Q=0.7 \mathrm{GeV})$ the $\mathrm{QCD}$ coupling approaches unity, the perturbative formulas certainly cease to be meaningful.
} 
represents only an extrapolation of twist-two QCD physics into the low $Q$ region-it is not intended to be a best fit. However, as demonstrated by the GRV parton distribution sets [32], this kind of extrapolation often turns out to compare rather well with data in the low $Q$ region. Comparison of CTEQ4LQ structure functions to the NMC, E665 and H1 data in the range $1.0<Q<3.0 \mathrm{GeV}$ is shown in Fig. 18. The parameters for CTEQ4D, CTEQ4L and CTEQ4LQ are also given in the Appendix.

The rather remarkably consistent picture resulting from this round of CTEQ4 global analysis incorporating jet data from hadron collisions provides a new generation of improved parton distributions for making calculations and predictions on high energy processes both within and beyond the standard model. The more tightly constrained parton distributions can also lay the foundation for more stringent tests of the pQCD framework and provide the basis for discerning signals of new physics.

At present, a remaining area of some uncertainty is the gluon distribution in the "large $x$ " region, beyond say 0.25 , where neither the DIS nor the direct photon data give tight constraints. For the DIS process, the sensitivity to the gluon begins below $x=0.1$. For the direct photon process there are a number of theoretical uncertainties which are not yet under control, as already discussed in the Introduction. The noticeable rise of the inclusive jet data points [7] above all "theory" curves shown so far may be related to the conventional choices of parametrization of the non-perturbative function $G\left(x, Q_{i}\right)$, which restricts its behavior in the large $x$ region. This possibility, first raised in Ref. [6], will be discussed next in the context of the CTEQ4 analysis presented above.

\section{High $E_{t}$ Jets and Parton Distributions}

The higher-than-expected inclusive jet cross-sections, first measured by the CDF collaboration [7] for $E_{t}>200 \mathrm{GeV}$, were observed in comparison to the existing parton distribution sets, including CTEQ3M as shown in Fig. 9. This "excess" is reduced slightly when jet data are included in the global fit, but is still noticeable in Figs. 13 and 14 for the CTEQ4A series of distributions. This is understandable since the high $E_{t}$ data points have large errors, so do not carry much statistical weight in the fitting process, and the simple (unsigned) $\chi^{2}$ is not sensitive to the observed pattern that all the points are higher than the theoretical prediction in the large $E_{t}$ region. Ref. [6] investigated the feasibility of accommodating these higher cross-sections in the conventional QCD framework by exploiting the flexibility of $G(x, Q)$ at higher values of $x$ where there are few independent constraints, while maintaining the agreement with other data sets in the global analysis. To do this, it is necessary to (i) provide enough flexibility in the parametrization of $G\left(x, Q_{0}\right)$ to allow for behaviors different from the usual (but arbitrary) choice; and (ii) focus on the high $E_{t}$ data points and assign them more statistical weight than their nominal values in order to force a better agreement 
between theory and experiment. Thus, the spirit of the investigation is not to obtain a "best fit" in the usual sense. Rather, it is (i) to find out whether such solutions exist; and (ii) if they do exist, to quantify how well these solutions agree with other data sets as compared to conventional parton distribution sets. The global analysis work described in Sec. 5 without special attention to the high $E_{t}$ points provides the natural setting to put the results of Ref. [6] in context.

Ref. [6] was performed using the CDF Run-IA data - the only high statistics inclusive jet measurement available at the time. Two illustrative "solutions" of the type described above were presented - one with the normalization fixed at 1.0 with respect to the CDF data, the other with a normalization factor of 0.93. Fig. 19 compares predictions of the normalization=1.0 PDF set, which we shall refer to as the CTEQ4HJ set, with the more recent Run-IB results of both $\mathrm{CDF}$ and D0. For this comparison, an overall normalization factor of 1.01(0.98) for the $\mathrm{CDF}(\mathrm{D} 0)$ data set is found to be optimal in bringing agreement between theory and experiment. 12 The consistency between the two data sets, as well as between theory and experiment, displayed by this comparison appears to be rather remarkable (again, bearing in mind the neglect of systematic errors other than overall normalization). Results shown in Table 4 quantify the $\chi^{2}$ values obtained while accommodating the high $E_{t}$ jets in the global fit in this particular case. Compared to the best fit CTEQ4M, the overall $\chi^{2}$ for CTEQ4HJ is indeed slightly higher. But this difference is much smaller than the differences discussed earlier in the CTEQ4A series, and much smaller than the difference between MRSJ and CTEQ4M. Thus the price for accommodating the high $E_{t}$ jets is negligible. In addition, the difference between CTEQ4HJ and CTEQ4M is almost entirely due to the BCDMS data, even though the BCDMS $\chi^{2}$ for CTEQ4HJ by itself is quite good. This change is due to the fact that, in the CTEQ4M fit, the BCDMS data set is the dominant one determining the large- $x$ quark distributions, while, in the CTEQ4HJ fit, the jet data set is in competition for these quark parameters, and they are changed by minute amounts. This is shown in Fig. 20 where the residuals between BCDMS data and theory are shown for CTEQ4M and CTEQ4HJ. The residuals are almost identical, which, together with Table \&, confirms the fact that even though CTEQ4HJ does not give the absolute overall best fit to all data, it provides an extremely good description of all data sets. It should be considered as a candidate for the gluon distribution in nature. ${ }^{3}$ In the future we will need strong, independent measurements of the large- $x$ gluons in order to clarify the situation with the high- $E_{t}$ jets.

\footnotetext{
${ }^{12}$ The change of CDF normalization factor from 1.0 to 1.01 is attributable to the switch from the Run-IA to the Run-IB data set.

${ }^{13}$ This is to be contrasted with the conclusion of incompatibility between the inclusive jet and DIS data reached by Ref. [33]. Their fit to inclusive jet data over the full $E_{t}$ range (the MRSJ' set) gives rise to an extremely large $\chi^{2}$ for the BCDMS data set.
} 


\section{Summary}

In this study of the impact of recent DIS and inclusive jet data on the global QCD analysis of lepton-hadron and hadron-hadron processes, we see significant progress in demonstrating the consistency of the NLO QCD framework, and in narrowing the uncertainties on the elusive but important gluon distribution. Specifically,

- The recent NMC, E665, H1 and ZEUS data considerably narrow down parton distributions and limit the behavior of the gluon, especially if one uses the minimal form of the gluon parameterization used by CTEQ3;

- The new inclusive jet data agree well with theory predictions based on PDF's determined by the other processes, with the possible exception of the high $E_{t}$ data points.

- By adding jet data to the global analysis, it is possible to further explore the range of variation of the gluon distribution using a more general parametrization. Although the jet data set covers a limited $x$-region, its effect is felt over the entire $x$-range - because it complements the other data sets well.

- Based on these investigations, a new generation of CTEQ4 parton distributions for a variety of features are presented: they are tabulated in Table 3.

- Three sources contributing to the uncertainty of the gluon distribution have been investigated: (i) by letting $\alpha_{s}$ vary over its current range of uncertainty; (ii) by increasing the degree of freedom for parametrizing the non-perturbative initial gluon distribution, and (iii) by varying the $Q_{c u t}$ in selecting data for the global fits. The largest effect is due to $\alpha_{s}$.

- These studies help to delineate the range of variation of $G(x, Q)$ over the range $10^{-4}<$ $x<0.25$. Further work is needed in exploring the range of uncertainty of the gluon and other parton distributions by systematically varying the relevant parameters of the global analysis.

- For larger values of $x$, more definitive experimental results on inclusive jet and direct photon production as well as improved theory are needed for further progress. The observed high $p_{t}$ "excess" jet cross-section can be accommodated by a modified gluon distribution, represented by the CTEQ4HJ set, since no other independent measurement constrains it in this range.

In view of the strong correlation between the gluon distribution and $\alpha_{s}$, narrowing the uncertainty in the latter will significantly improve the determination of $G(x, Q)$. What can a global analysis of experimental data described in this paper contribute to the measurement 
of $\alpha_{s}$ ? To explore this question, one needs to study in some detail the sensitivity of each process which contributes to the global analysis to the variation of $\alpha_{s}$. This problem will be pursued in a separate analysis.

\section{Appendix}

\section{CTEQ4 Parton Distribution Parameters}

The initial parton distributions at $Q=Q_{0}, f^{i}\left(x, Q_{0}\right)$, are parametrized in general as in Eq. 3 for the gluon $G$ and the quark flavors $d_{v}, u_{v}, \bar{u}+\bar{d}, s(\bar{s})$; except for the combination $\bar{d}-\bar{u}$ (which does not have to be positive definite) which is parametrized as:

$$
\bar{d}-\bar{u}=A_{0} x^{A_{1}}(1-x)^{A_{2}}\left(1+A_{3} \sqrt{x}+A_{4} x\right)
$$

For all parton distribution sets, $Q_{0}=1.6 \mathrm{GeV}$, except for CTEQ4LQ which has $Q_{0}=0.7$ $\mathrm{GeV}$. Tables of the coefficients $\left\{A_{n}^{i} ; n=1, . ., 4 ; i=\right.$ flavors $\}$ for the three standard parton distribution sets CTEQ4M, CTEQ4D, CTEQ4L and the low- $Q_{0}$ set CTEQ4LQ are given below, in Tables 6,7, 8 and 9. All parton distribution sets listed in Table 3 are available in fortran program form by request $\square^{-1}$ or via WWW at http://www.phys.psu.edu/cteq/.

\section{Experimental Normalization Factors}

The $\chi^{2}$ tables 4,5 are obtained by allowing the experimental data sets to "float" with respect to the theory cross-sections. For CTEQ distributions, a $\chi^{2}$ penalty is included in the fitting process for deviations of the normalization factors with respect to the respective overall experimental normalization errors. For non-CTEQ distributions, we simply obtained the minimum $\chi^{2}$ by varying the normalization factors without such penalty. The resulting normalization factors which go with Tables 1,5 are given in Tables 10 and 11 .

\section{Experimental Systematic Uncertainties}

For DIS, DY and direct photon data, we follow the usual procedure of combining in quadrature point-to-point systematic errors given by the experiments with the statistical errors. Correlated systematic errors other than overall normalization are not generally available from most experiments. For a few where they are, we have done separate studies of the consequences of incorporating them in the global analysis and found they do not affect the best fit parameters by any significant amount. See Ref. 13.

For the preliminary inclusive jet data, only the normalization uncertainty is taken into account in the global fit. The rationale has been explained in Sec. 5. The fully correlated

\footnotetext{
${ }^{14}$ Requests can be sent to Lai_H@Pa.Msu.Edu or Tung@Pa.Msu.Edu.
} 
systematic errors from CDF, although available, are not easily implemented in a way which is consistent with all the other data sets. (A separate study on the effects of these uncertainties employing the full correlation matrix is underway, and it will be reported in the future.) These errors are not yet available for the D0 data set. The main effect of omitting the systematic errors on jets is to increase somewhat the relative weight of this data set in the global analysis. This will not affect the fits substantially because the jet data agree well with parton distributions determined from other processes, as discussed in Sec. 4 .

In general, the question of assigning appropriate relative weights to different experimental data sets in a global analysis is a difficult one. An experiment with few data points which is however particularly sensitive to some physical parameters than all the others can sometimes be emphasized justifiably in a global $\chi^{2}$ minimization process, otherwise it will be overwhelmed by the far more numerous data sets and the sensitivity will be lost. As an extreme example, the NA51 experiment [29], which has an important impact on the determination of the flavor $\mathrm{SU}(2)$ assymmetry of the sea quarks $(\bar{u}-\bar{d})$, consists of only one data point. It has to be appropriately emphasized in a global analysis to have an effect in differentiating the sea quarks.

\section{Dependence on the Choice of $Q_{c u t}$}

In all global QCD studies, a set of cut-offs on the hard scale "Q" for various processes is used in data selection. In recent CTEQ analyses, this $Q_{\text {cut }}$ has been $2 \mathrm{GeV}$ on $Q$ and $3.5 \mathrm{GeV}$ on $W$ in DIS, $2 \mathrm{GeV}$ on $Q$ (the invariant lepton pair mass) in Drell-Yan process, and $4 \mathrm{GeV}$ on $p_{t}$ in direct photon production. As a final check on the reliability of the results described in the previous section, we test the sensitivity of the fits to the value of these cut-offs in order to gauge possible influence due to non-perturbative or higher-twist effects.' ${ }^{\text {m }}$ For this purpose, we carried out several series of analyses similar to the CTEQ4A-series above, but with the minimum $Q_{\text {cut }}$ raised progressively from $2 \mathrm{GeV}$ to 3 , 4, and $5 \mathrm{GeV}$. Data points excluded by these higher $Q_{c u t}$ 's are mainly those of fixed-target DIS experiments. We found our results to be rather stable under these changes. Fig. 21 compares the gluon distributions from three PDF sets obtained with three $Q_{c u t}$ values mentioned above, all for a given $\alpha_{s}$ value of 0.113 . We see that the differences are quite small - smaller than those due to the variation of $\alpha_{s}$ (with the same $Q_{c u t}$ ) shown in Fig. 14 and described in Sec. 5. The subtle differences, especially in relation to sensitivity on $\alpha_{s}$ values, will be discussed elsewhere [16].

\footnotetext{
${ }^{15}$ This issue has previously been investigated in Ref. [34. The accuracy of both experiments and theory have improved dramatically since then.
} 


\section{Acknowledgement}

We would like to thank our CTEQ colleagues, R. Brock, J. Collins, J. Morfin, J. Pumplin, J.W. Qiu, J. Smith, G. Sterman, J. Whitmore, and C.-P. Yuan, for very useful discussions and encouragement.

\section{References}

[1] G. Sterman et al., Handbook of Perturbative QCD, Rev. Mod. Phys. 67, 157 (1995).

[2] For a review and detailed references, see J.F. Owens and W.K. Tung, "Parton Distributions of Hadrons" in Ann. Rev. Nucl. Sci. 42, 291 (1992).

[3] WA70: M. Bonesini et al., Z. Phys. C38, 371 (1988).

[4] CTEQ Collaboration: J. Huston et al., Phys. Rev. D 51, 6139 (1995).

[5] E.L. Berger, X. Guo, J. Qiu, Phys. Rev. D53, 1124 (1996); and ANL-HEP-PR-95-88, hep-ph/9512281

[6] CTEQ Collaboration: J. Huston et al., MSUHEP-50812, e-Print Archive: hepph/9511386, to appear in Phys. Rev. Lett.

[7] CDF Collaboration Run-IA: (Abe et al.), Fermilab-PUB-96/020-E (1996) to appear in Phys. Rev. Lett.; and Run-IB: B. Flaugher, Talk given at APS meeting, Indianapolis, May, 1996.

[8] D0 Collaboration: G. Blazey, Talk given at Rencontre de Moriond, March, 1996; D. Elvira, Talk given at Rome conference on DIS and Related Phenomena, April, 1996.

[9] S. Ellis, Z. Kunszt, and D. Soper, Phys. Rev. Lett. 642121 (1990); S. Ellis, Z. Kunszt, and D. Soper, Phys. Rev. Lett. 69, 3615 (1992).

[10] F. Aversa et al., Phys. Rev. Lett. 65, 401 (1990).

[11] W. Giele et al., Nucl. Phys. B403, 2121 (1993).

[12] See Refs. [9], [10] and [1].

[13] CTEQ Collaboration: H.L. Lai et al., Phys. Rev. D 51, 4763 (1995).

[14] See, for example, Particle Data Group ( L. Montanet et al), Review of Particle Properties, Phys. Rev. D50, 1173 (1994); S. Bethke, p. 213, 30th Rencontres de Moriond: QCD and High Energy Hadronic Interactions, . Ed. J. Trân Thanh Vân. Editions Frontieres (1995). 
[15] Some related papers use a wider range of $\alpha_{s}$, extending up to 0.125 or 0.130 . Such large values are in strong disagreement with the highly precise BCDMS and CCFR experiments. Specifically, in a typical global fit involving around 1000 data points, the $\chi^{2}$ value increases by about 200 above the best fit, mostly from these two experiments.

[16] H.L. Lai et al., MSUHEP-60601, CTEQ610.

[17] A.D. Martin, R.G. Roberts, W.J. Stirling, Phys. Rev. D 506734 (1994); Phys. Lett. B354 155 (1995).

[18] NMC Collaboration: (M. Arneodo et al.) Phys. Lett. B364, 107 (1995).

[19] H1 Collaboration (S. Aid et al.): "1993 data" Nucl. Phys. B439, 471 (1995); "1994 data", DESY-96-039, e-Print Archive: hep-ex/9603004

[20] ZEUS Collaboration (M. Derrick et al.): "1993 data" Z. Phys. C65, 379 (1995) ; "1994 data", DESY-96-076 (1996).

[21] E665 Collaboration (M.R. Adams et al.): FNAL-Pub-95/396-E, 1996.

[22] G. Sterman and G. Korchemsky, Nucl. Phys. B437, 415 (1995); R. Akhoury and V.I. Zakharov, Nucl.Phys. B465, 295 (1996); Yu.L. Dokshitser, G. Marchesini, B.R. Webber, CERN-TH-95-281, e-Print Archive: hep-ph/9512336

[23] H.L. Lai and W.K. Tung, "Comparison of CDF and D0 Inclusive Jet Cross-sections", MSU-HEP-60508, CTEQ-605, e-Print Archive: hep-ph/9605269.

[24] See, for example; J. Ellis, D. A. Ross. CERN-TH-96-108, e-Print Archive: hepph/9604432; V. Barger, K. Cheung, P. Langacker, MADPH-96-936, e-Print Archive: hep-ph/9604298; J. Lykken, FERMILAB-PUB-96-070-T, e-Print Archive: hepth/9603133; R.S. Chivukula, A.G. Cohen, E.H. Simmons, BUHEP-96-5, e-Print Archive: hep-ph/9603311.

[25] The set with $\alpha_{s}=0.105$ is not included here and in subsequent comparisons because it is strongly disfavored by the new H1 and ZEUS data included in the fit to the B- and Cseries, by the inclusive jet data used in the CTEQ4 fits, and by the LEP measurements of $\alpha_{s}$ - even if the total $\chi^{2}$ of the $\alpha_{s}=0.105$ fit on DIS and DY data sets by themselves is comparable to that of the $\alpha_{s}=0.122$ set.

[26] BCDMS Collaboration (A.C. Benvenuti, et.al..), Phys.Lett. B223, 485 (1989); and Phys. Lett.B237, 592 (1990).

[27] CCFR Collaboration (W.C. Leung, et al.), Phys. Lett. B317, 655 (1993); and (P.Z. Quintas, et al.), Phys. Rev. Lett. 71, 1307 (1993). 
[28] E605: (G. Moreno, et al.), Phys. Rev. D43, 2815 (1991).

[29] NA51 Collaboration (A. Baldit, et al.), Phys. Lett. B332, 244 (1994).

[30] CDF Collaboration (F. Abe et al.), Phys. Rev. Lett. 74, 850 (1995).

[31] UA6 Collaboration ( G. Sozzi et al), Phys. Lett. B317, 243 (1993).

[32] M. Gluck, E. Reya, A. Vogt, Z. Phys. C67, 433 (1995).

[33] E.W.N. Glover, et.al. e-Print Archive: hep-ph/9603327.

[34] J.G. Morfin and W.K. Tung, Z. Phys. C52, 13 (1991). 

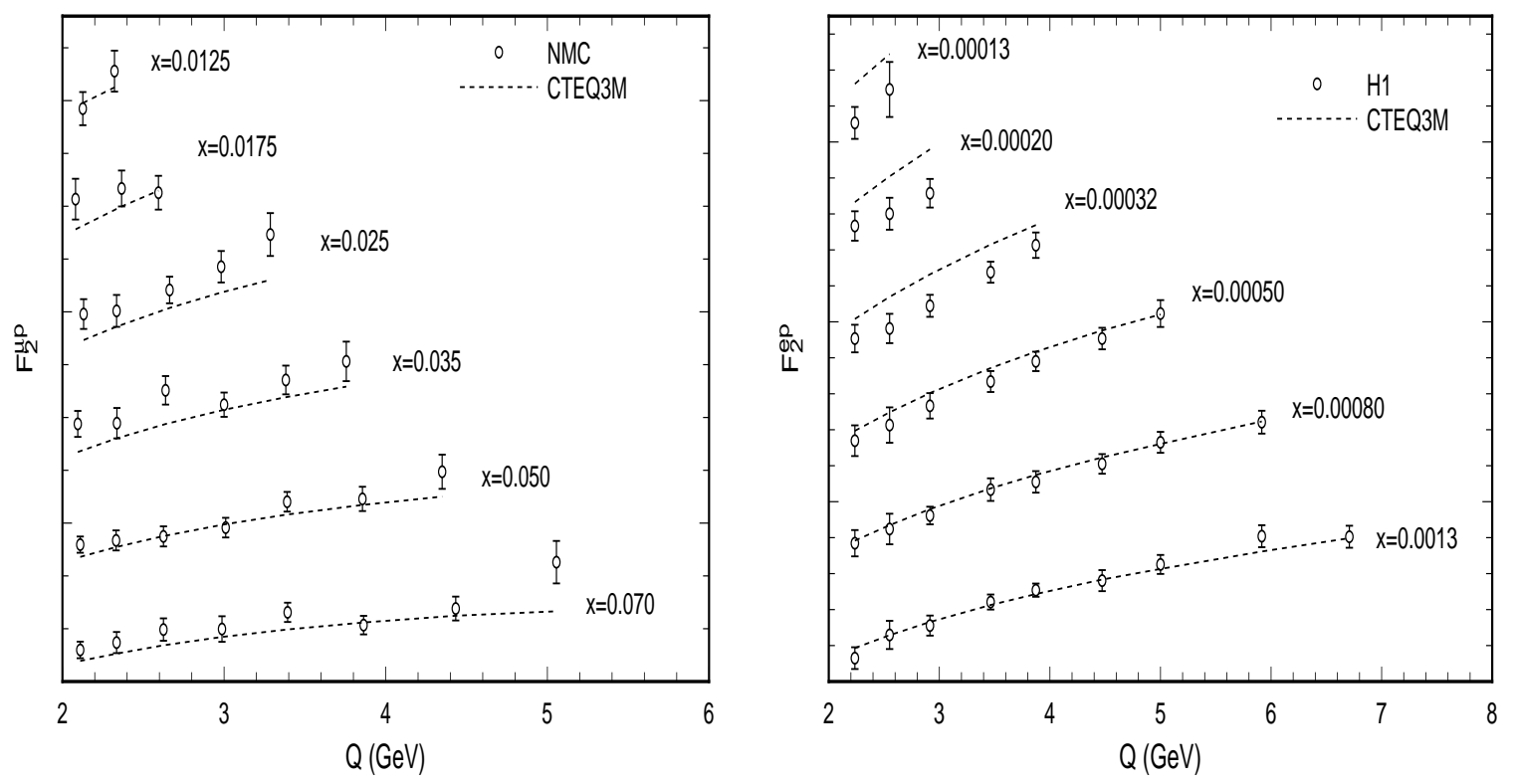

Figure 1 : Comparison of NLO calculations based on the previous generation CTEQ3M parton distributions with the latest NMC (a) and H1 (b) data in the small- $x$ region where discrepencies appear. 


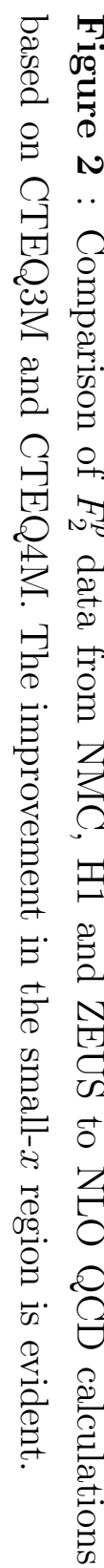
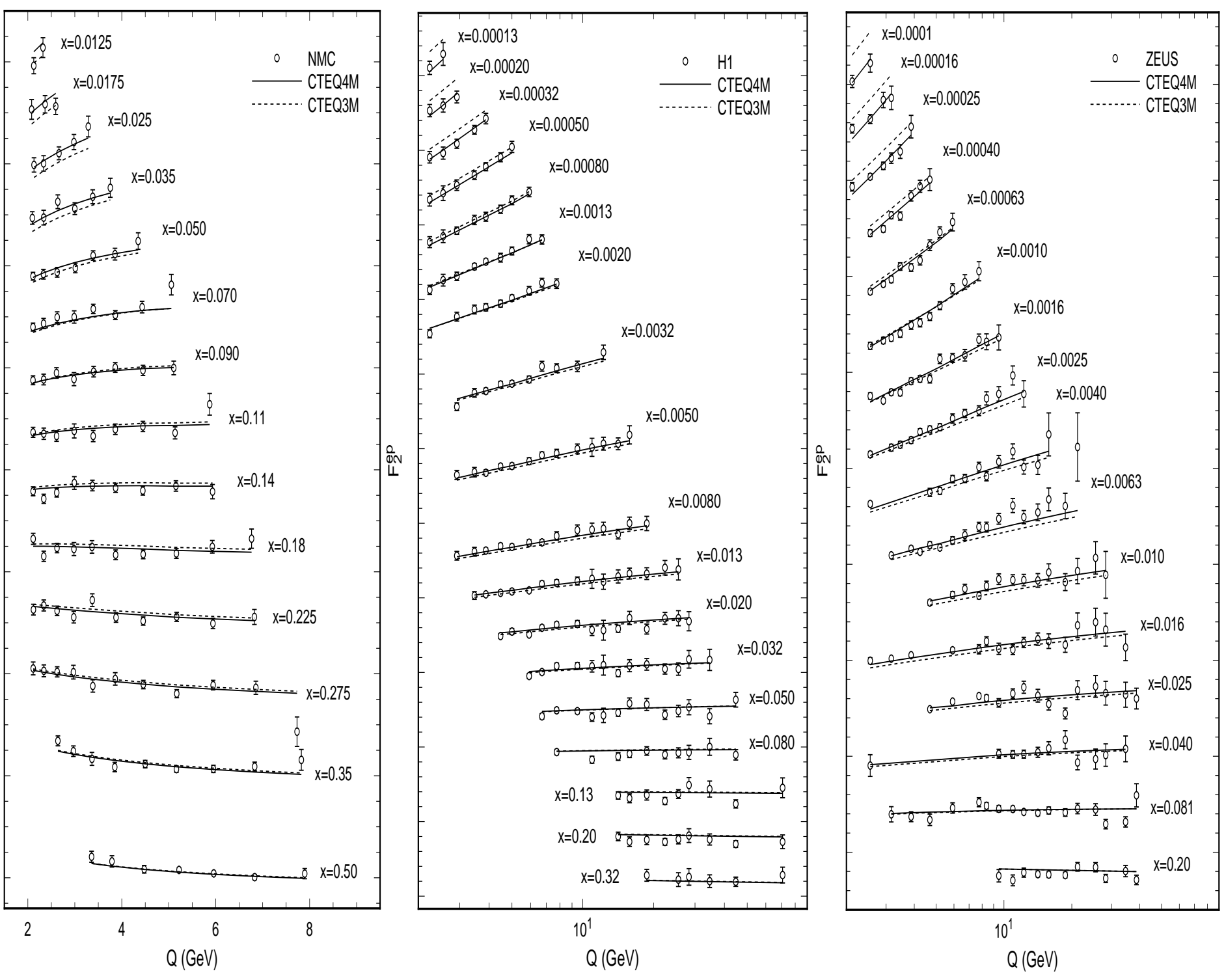

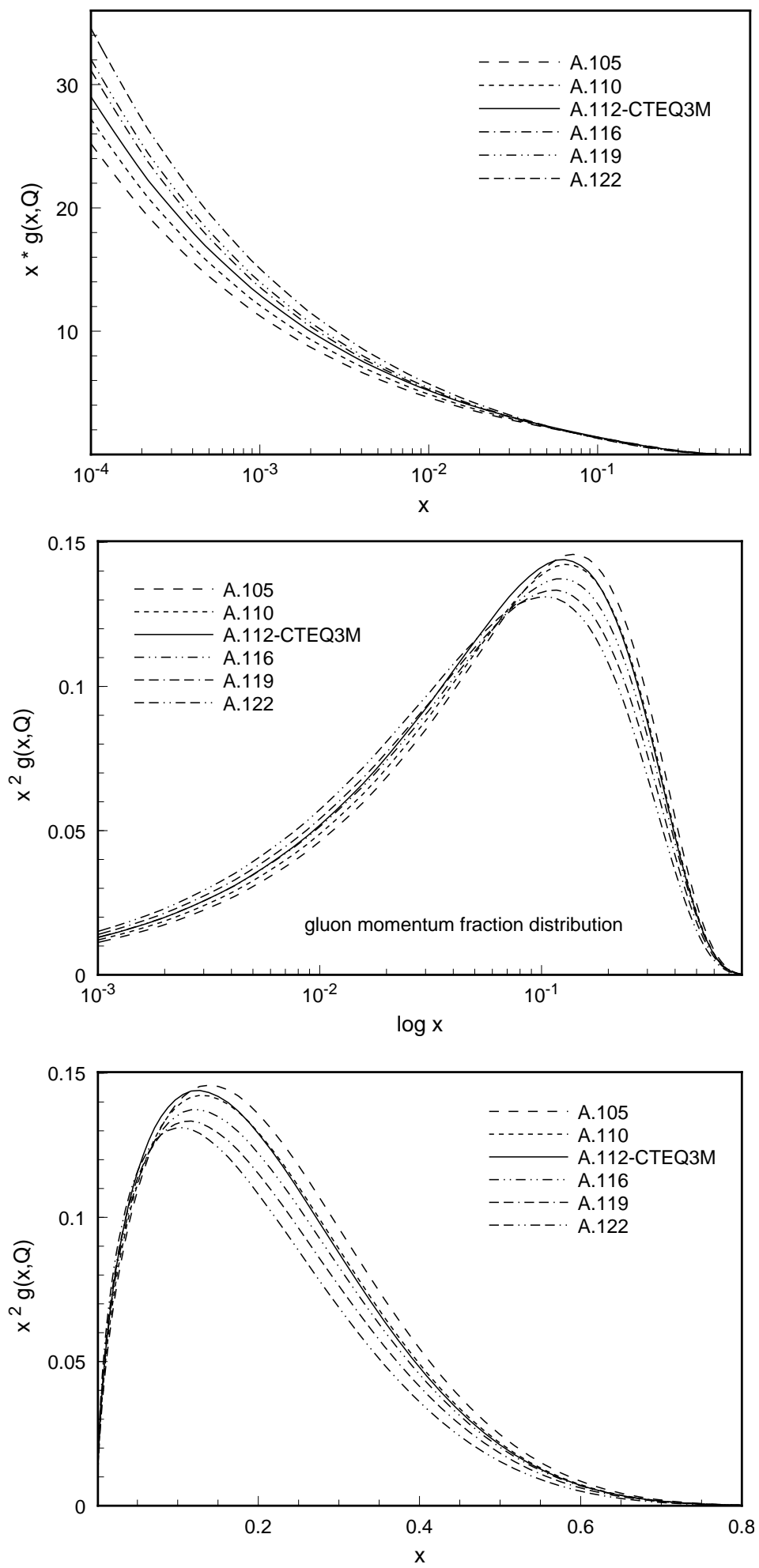

Figure 3 : Series-A gluon distributions in the small-, medium-, and large- $x$ regions. A.105 refers to the gluon associated with $\alpha_{s}\left(M_{Z}\right)=0.105$, and likewise for the other ones. 


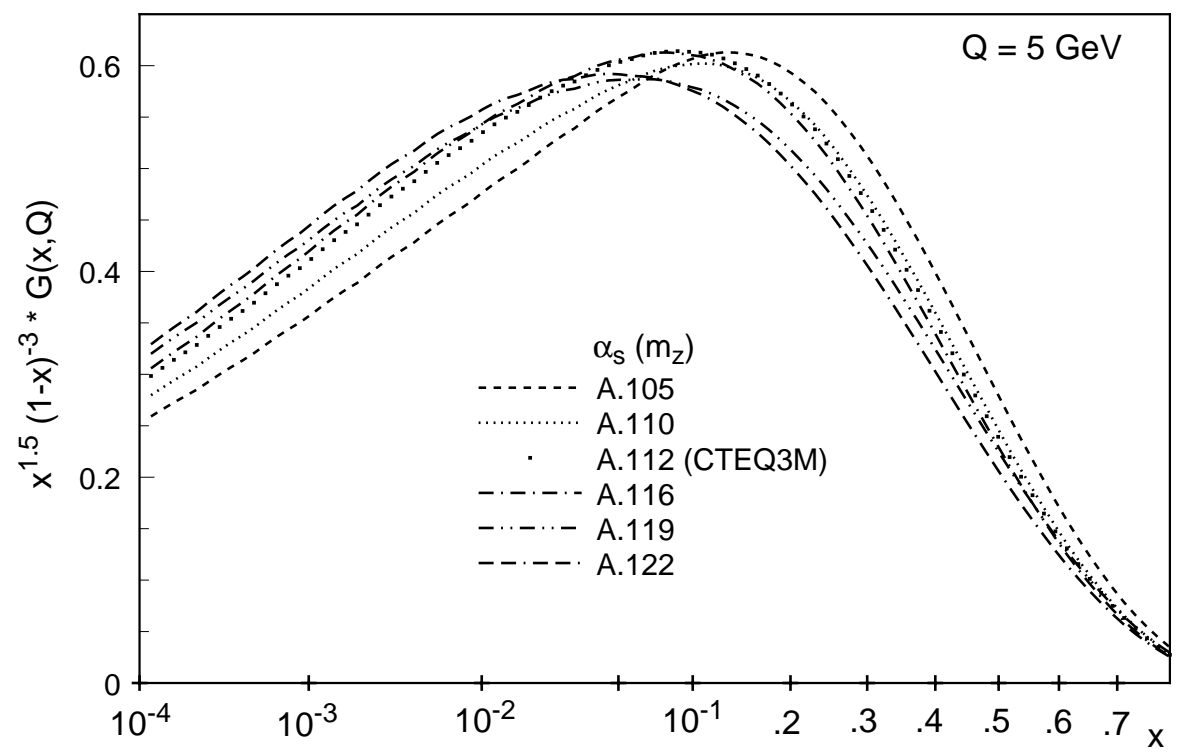

Figure 4 : Series-A gluon distributions normalized by the function $x^{-1.5}(1-x)^{3}$ in order to display clearly the behavior of $G(x, Q)$ over the entire $x$-range. For the same purpose, the horizontal $\mathrm{x}$-axis is drawn with a scale which smoothly changes from log- to linear behavior.

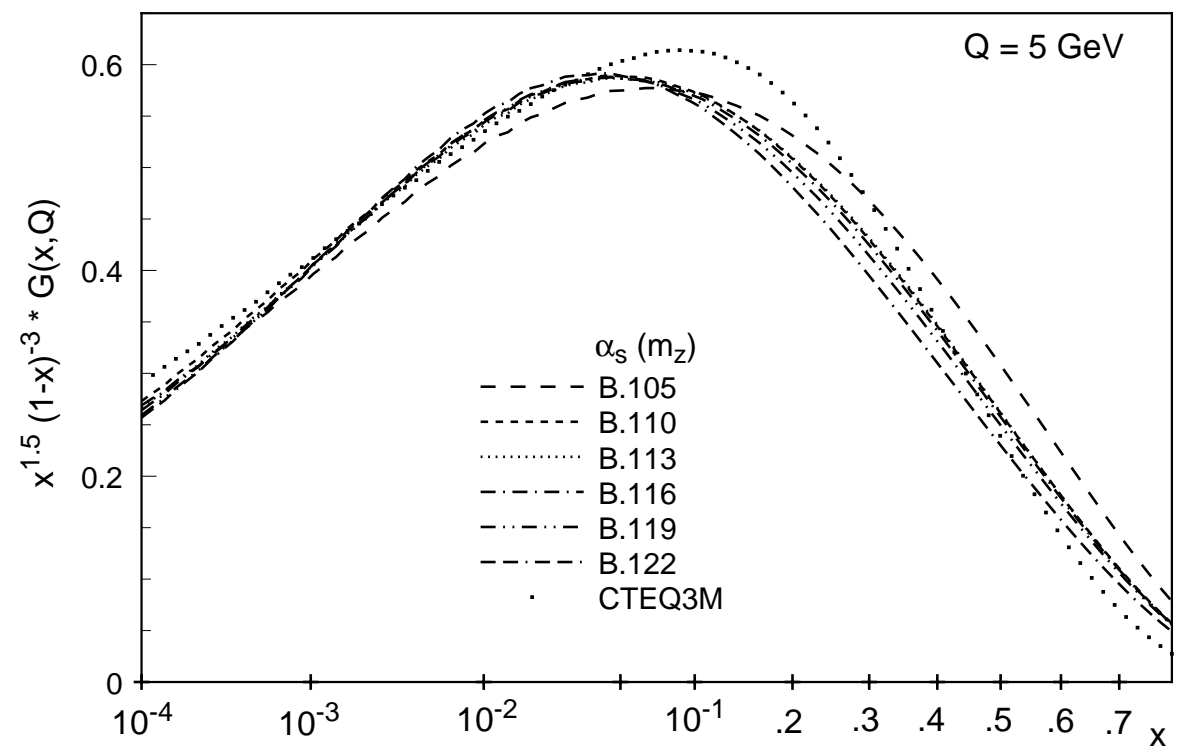

Figure 5 : Series-B gluon distributions normalized by the function $x^{-1.5}(1-x)^{3}$ (cf. caption of previous figure.) 


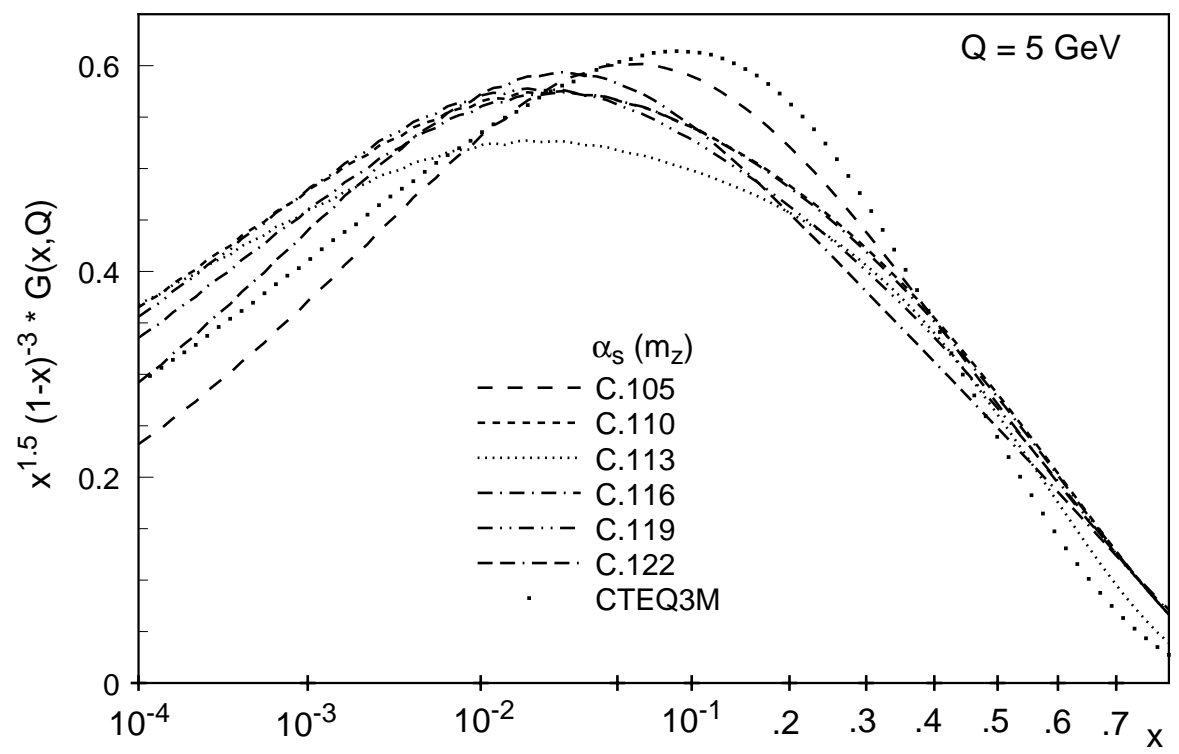

Figure 6 : Series-C gluon distributions normalized by the function $x^{-1.5}(1-x)^{3}$

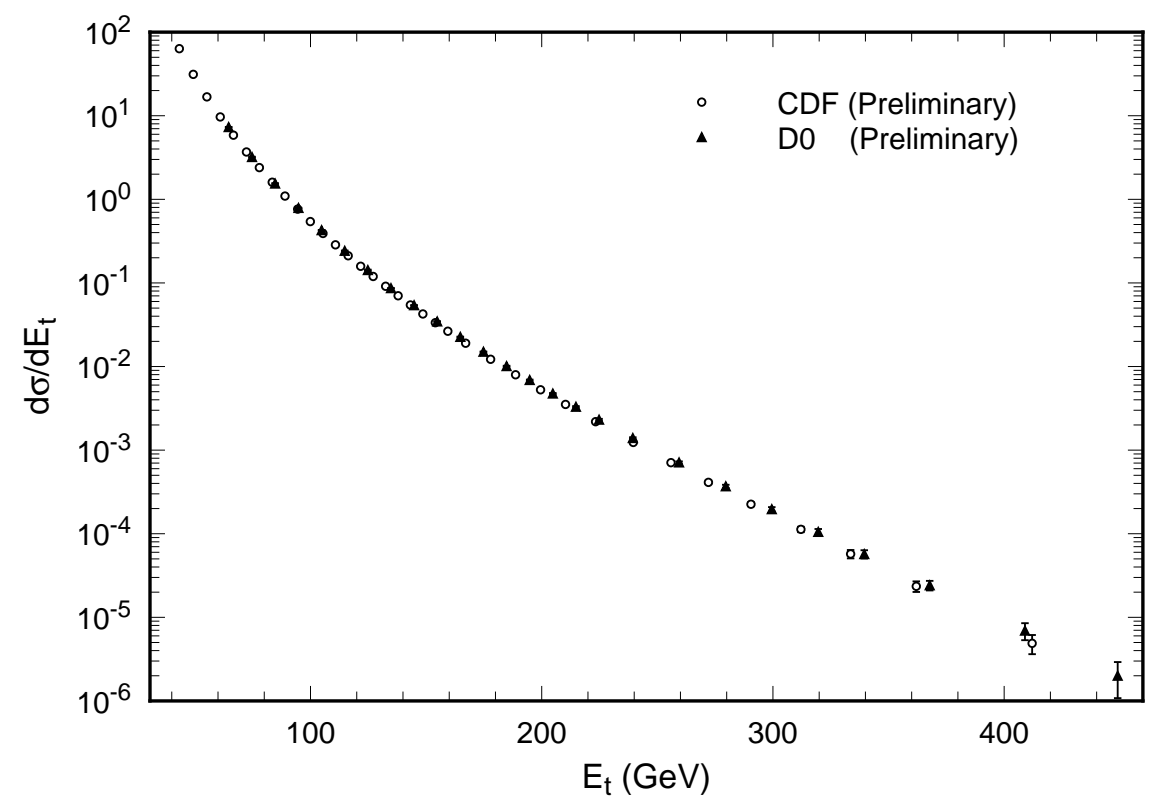

Figure 7 : Inclusive jet cross-section measured by the CDF and D0 collaborations in RunIB at the Tevatron. (Averaged over $0.1<|\eta|<0.7$ in the case of CDF and $|\eta|<0.5$ in the case of D0.) 

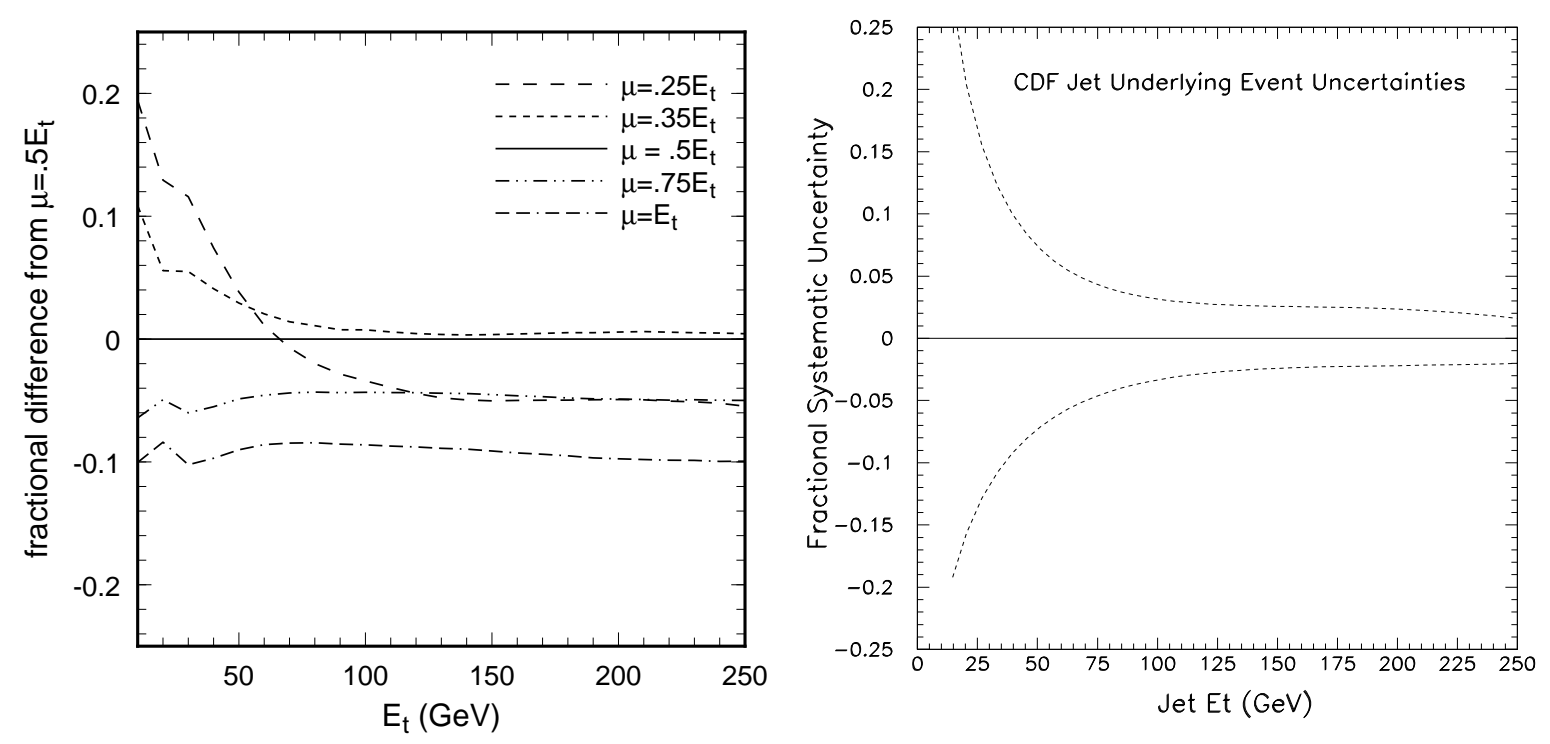

Figure 8 : Two examples of sources of uncertainties in comparing inclusive jet data with NLO QCD theory: (a) Fractional difference between $d \sigma\left(E_{t}, \mu\right) / d E_{t}$ and $d \sigma\left(E_{t}, \mu=E_{t} / 2\right) / d E_{t}$ (for the CDF rapidity coverage $0.1<|\eta|<0.7$ ) as a function of $E_{t}$ for a variety values of $\mu$; (b) Fractional change in the cross-section due to $\pm 30 \%$ change in underlying event correction in the CDF experiment.

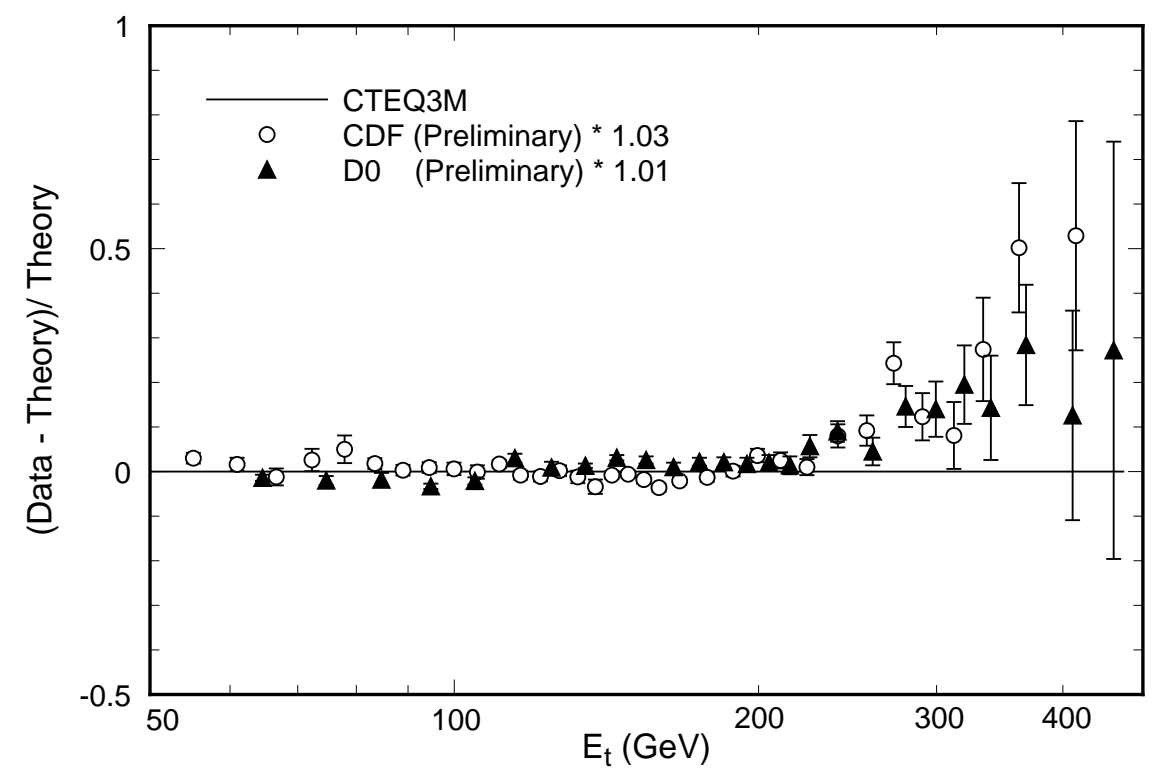

Figure 9 : Inclusive jet cross-section measured by the CDF and D0 collaborations in RunIB at the Tevatron normalized to NLO QCD calculations based on CTEQ3M PDF's. The difference in rapidity coverage of the two experiments is taken into account. 


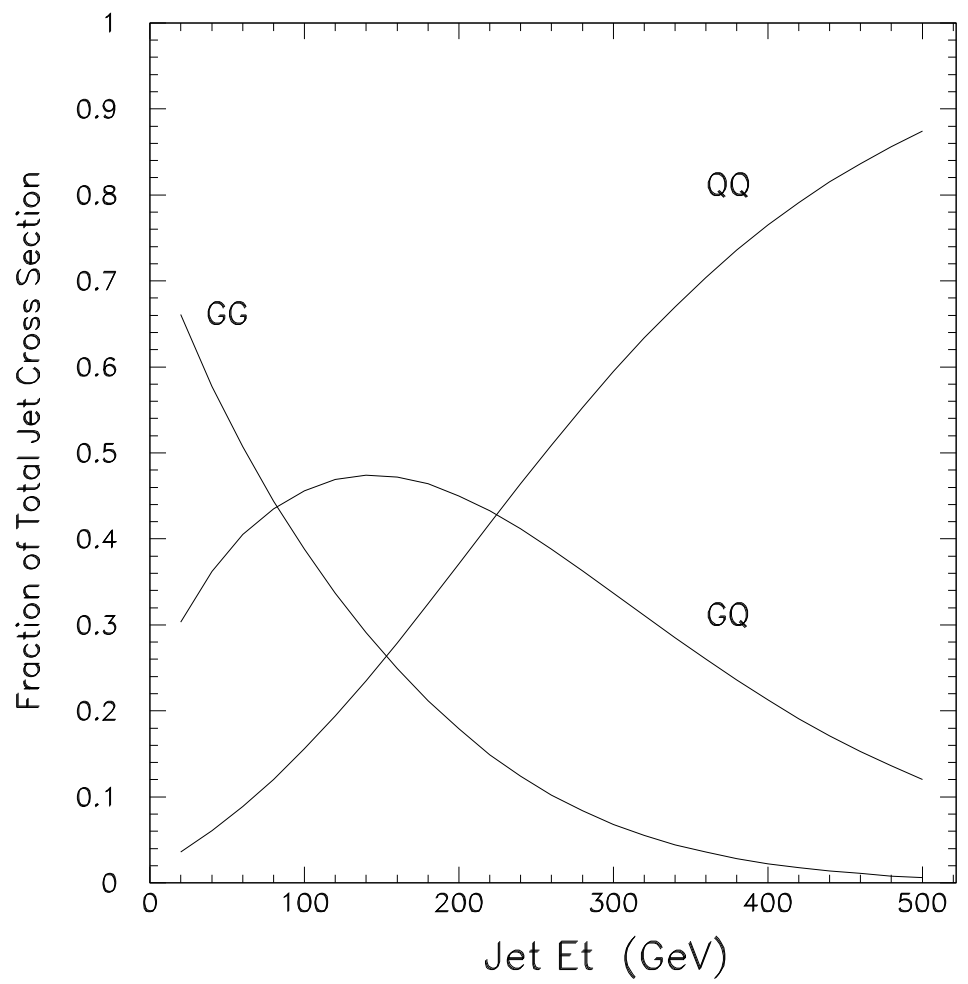

Figure 10 : Relative contribution to the inclusive jet cross-section due to the various partonic subprocesses.

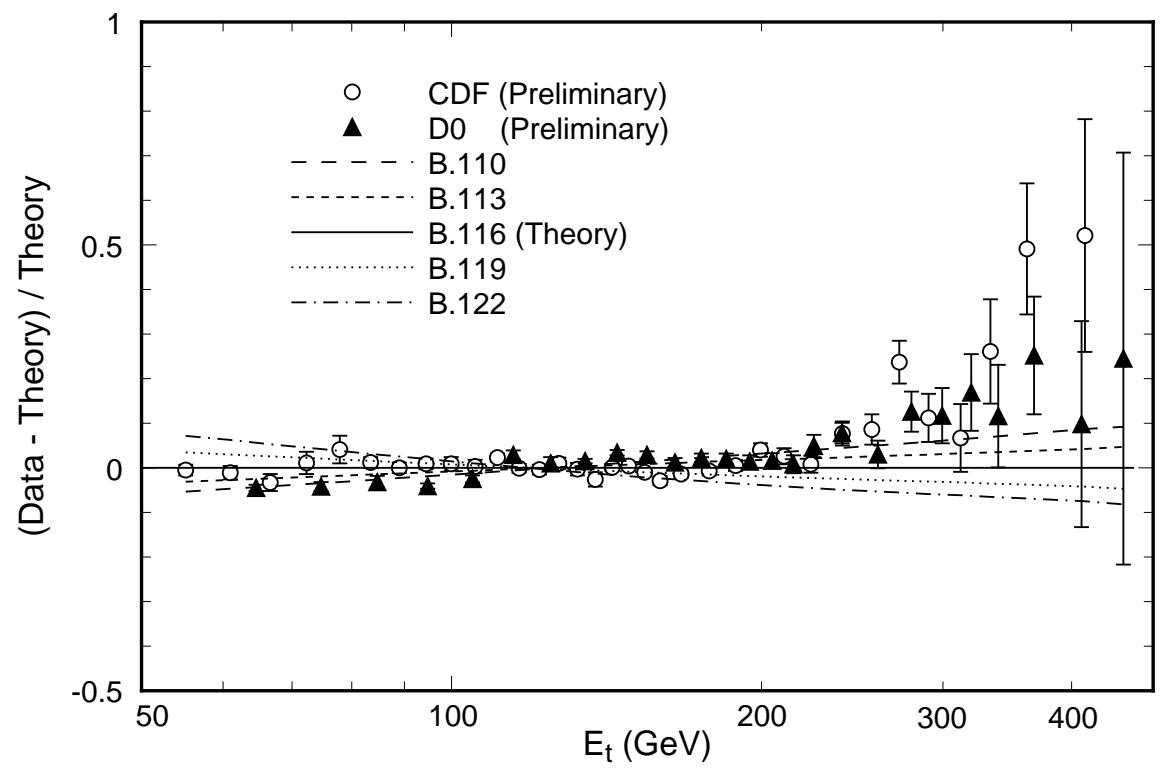

Figure 11 : Inclusive jet cross-section of CDF and D0 compared to NLO QCD calculations based on the new B-series parton distributions. 


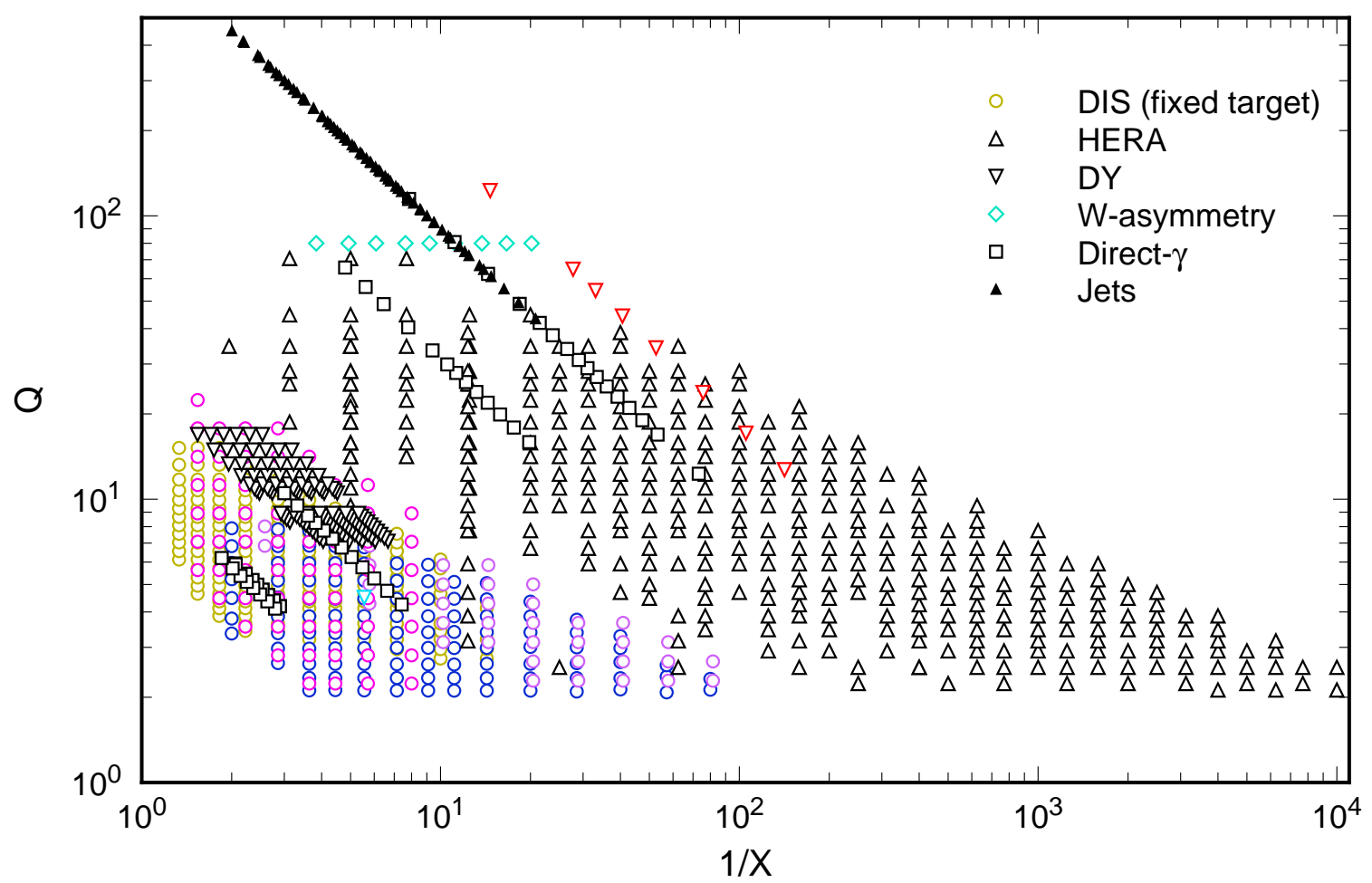

Figure 12 : Kinematic map in the $(x, Q)$ plane of data points used in the current global analysis.

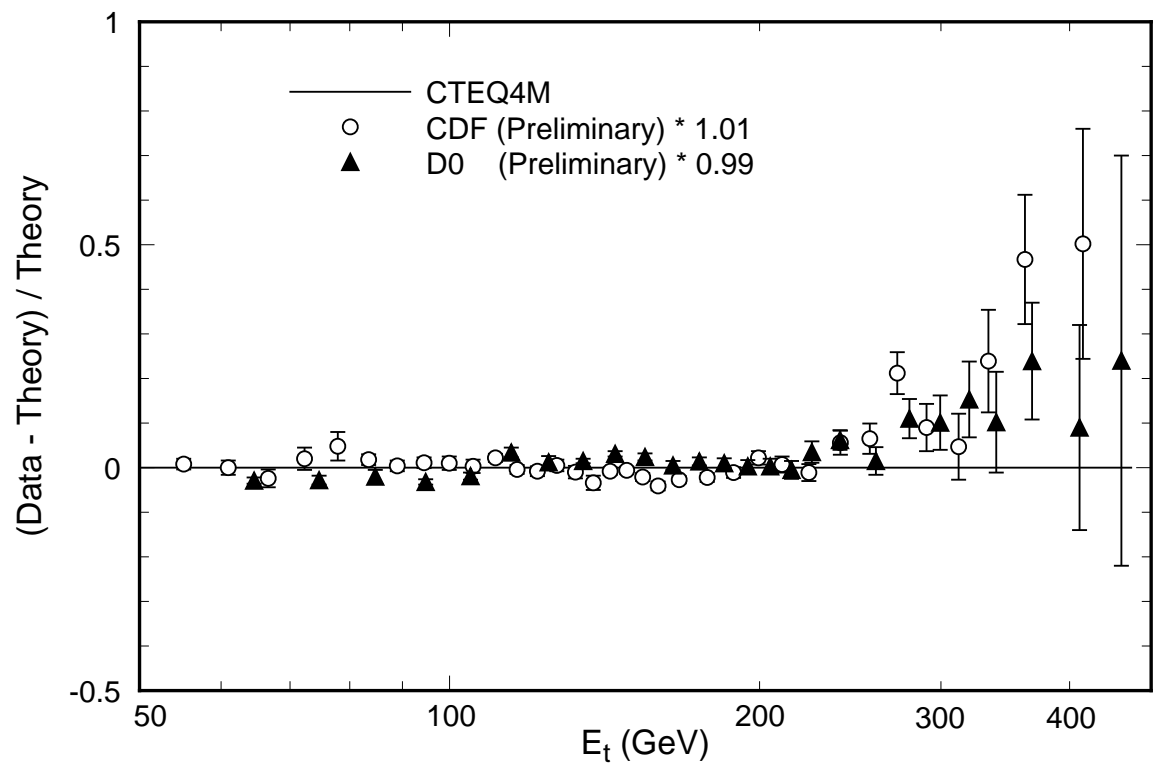

Figure 13 : Inclusive jet cross-section of CDF and D0 compared to NLO QCD calculations based on the new CTEQ4M parton distributions. 


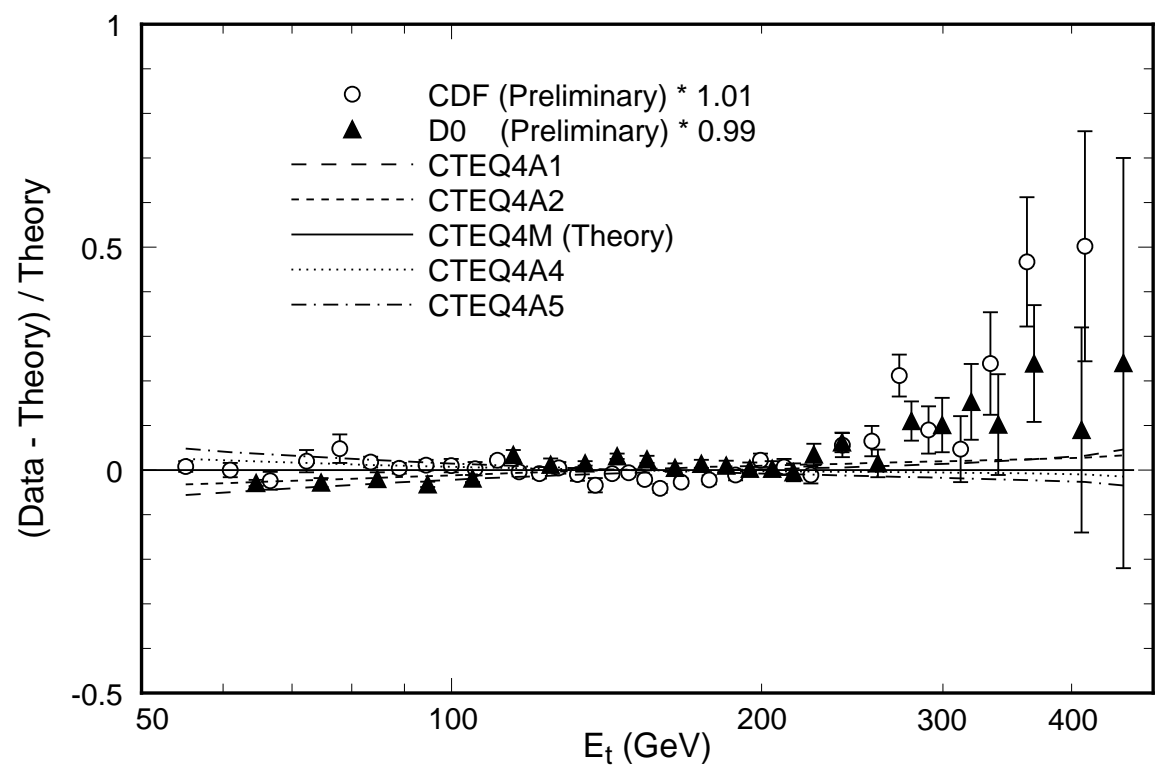

Figure 14 : Inclusive jet cross-section of CDF and D0 compared to NLO QCD calculations based on the new CTEQ4A series of parton distributions.

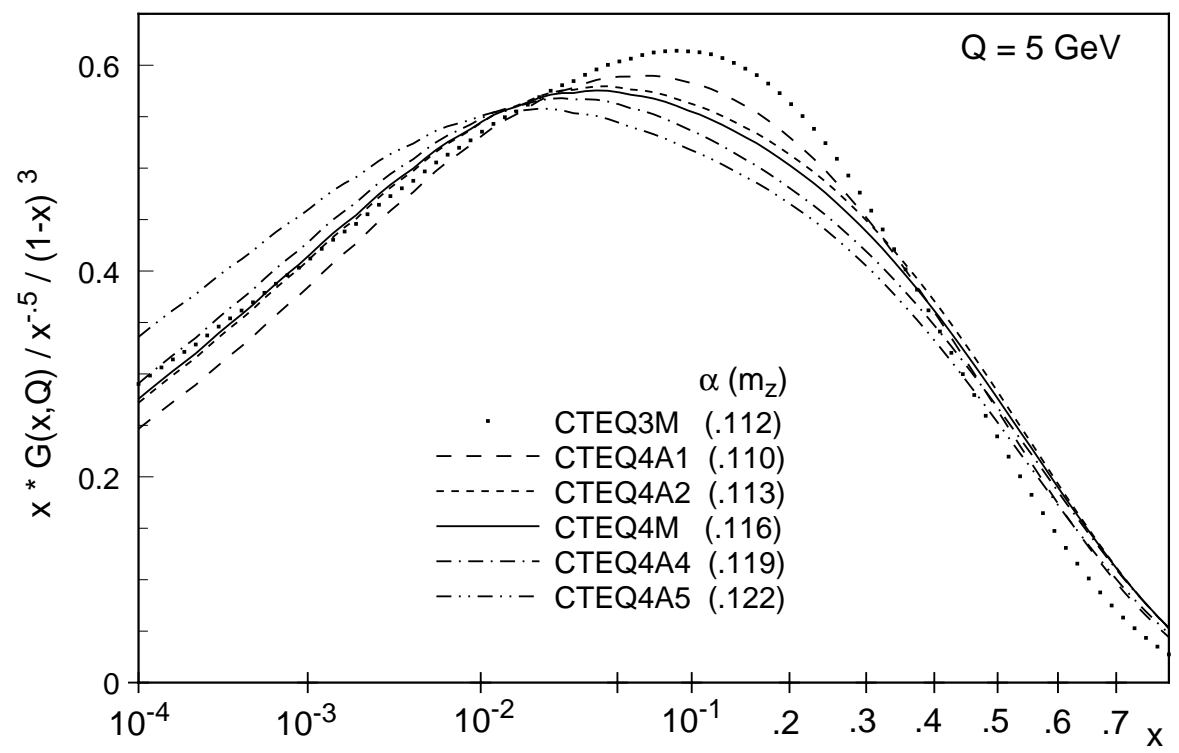

Figure 15 : Series-CTEQ4A gluon distributions normalized by the function $x^{-1.5}(1-x)^{3}$. 


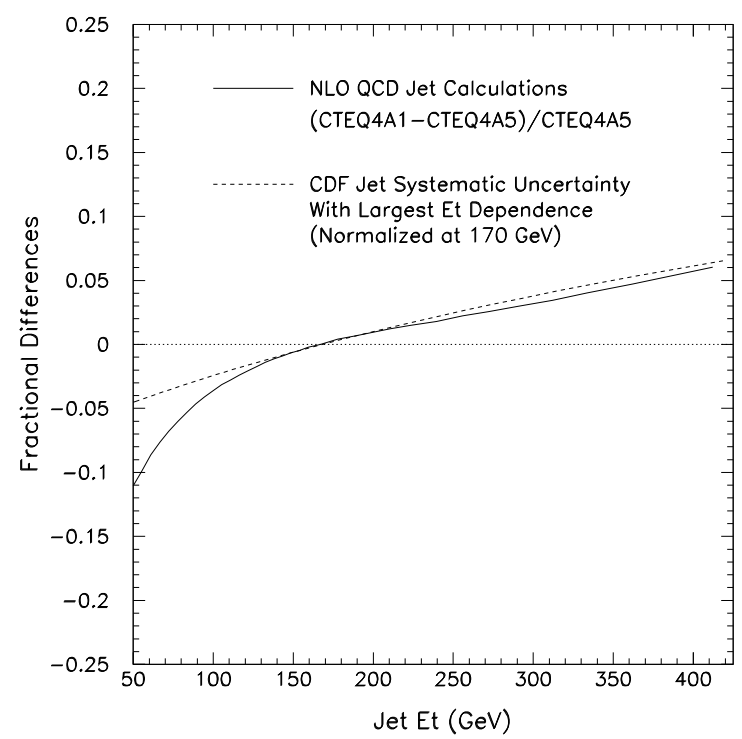

Figure 16 : Percentage range of variation of the inclusive jet cross-section from the two extreme CTEQ4A PDF sets (CTEQ4A1 and CTEQ4A5) compared to the largest of the $E_{t}$ dependent systematic uncertainties.

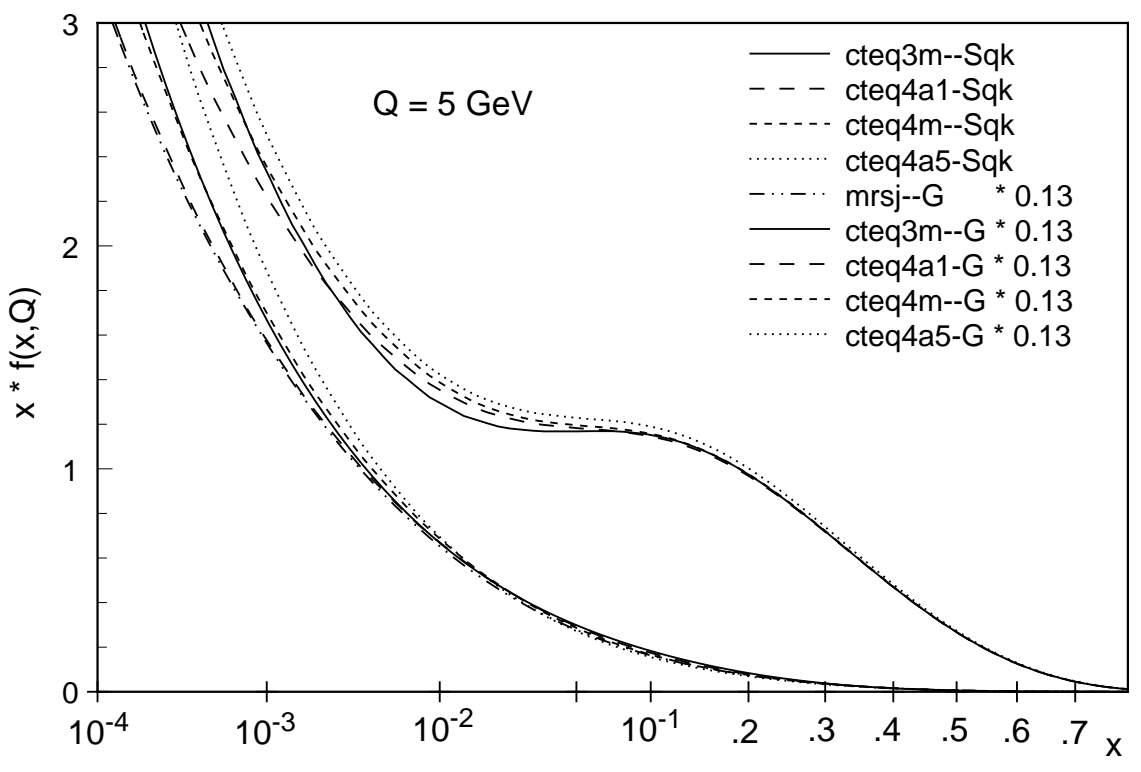

Figure 17 : Comparison of $x G(x, Q)$ and $x S(x, Q)$ between some new parton distribution sets and those from CTEQ3M. $S(x, Q)$ is the singlet quark distribution (sum over all flavors). The CTEQ3M and CTEQ4M gluons appear to lie on top of each other. The same is true for the CTEQ4A1 and MRSJ gluons. Differences in $G(x, Q)$ for $x>0.01$ are not evident in this plot. 

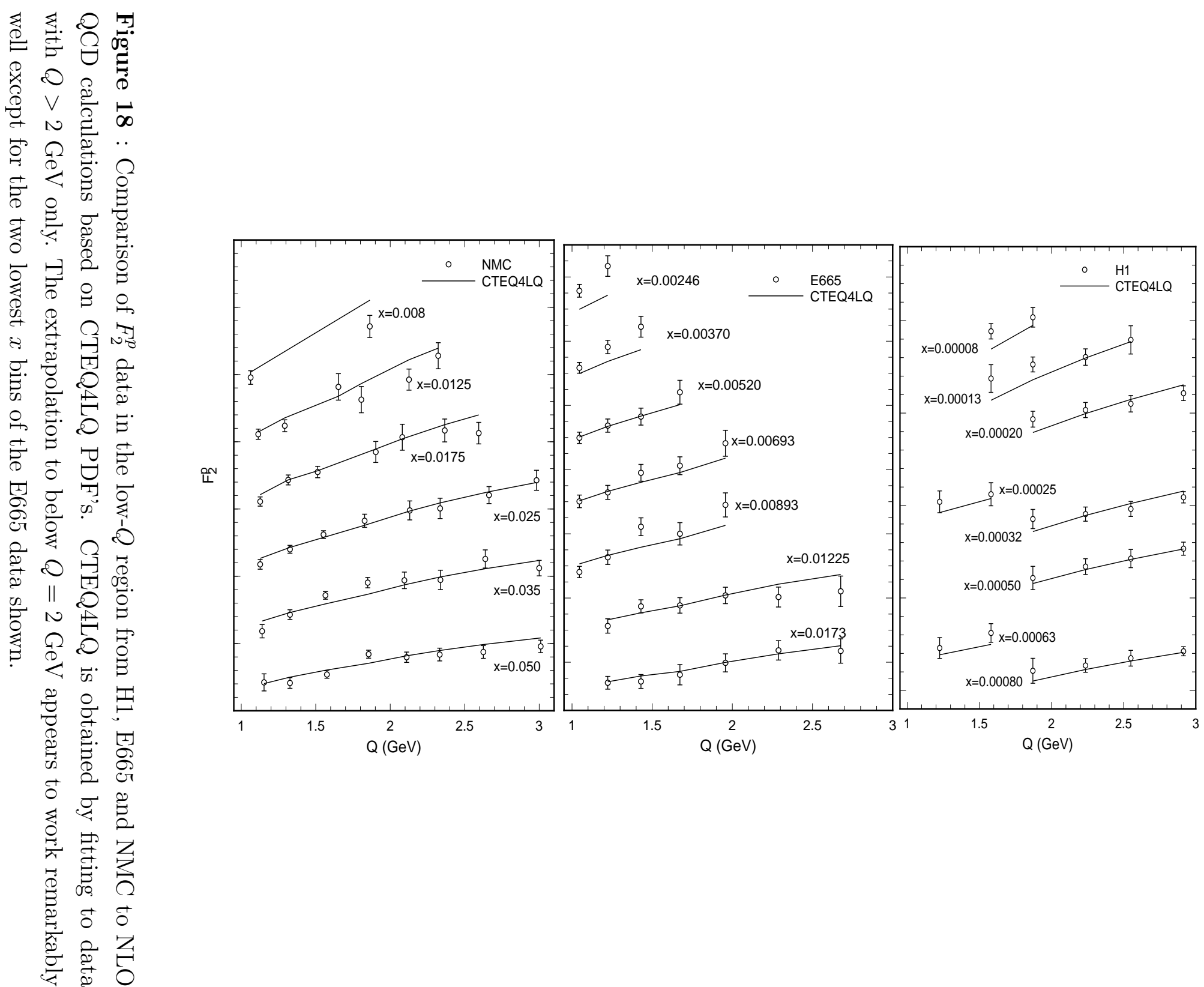


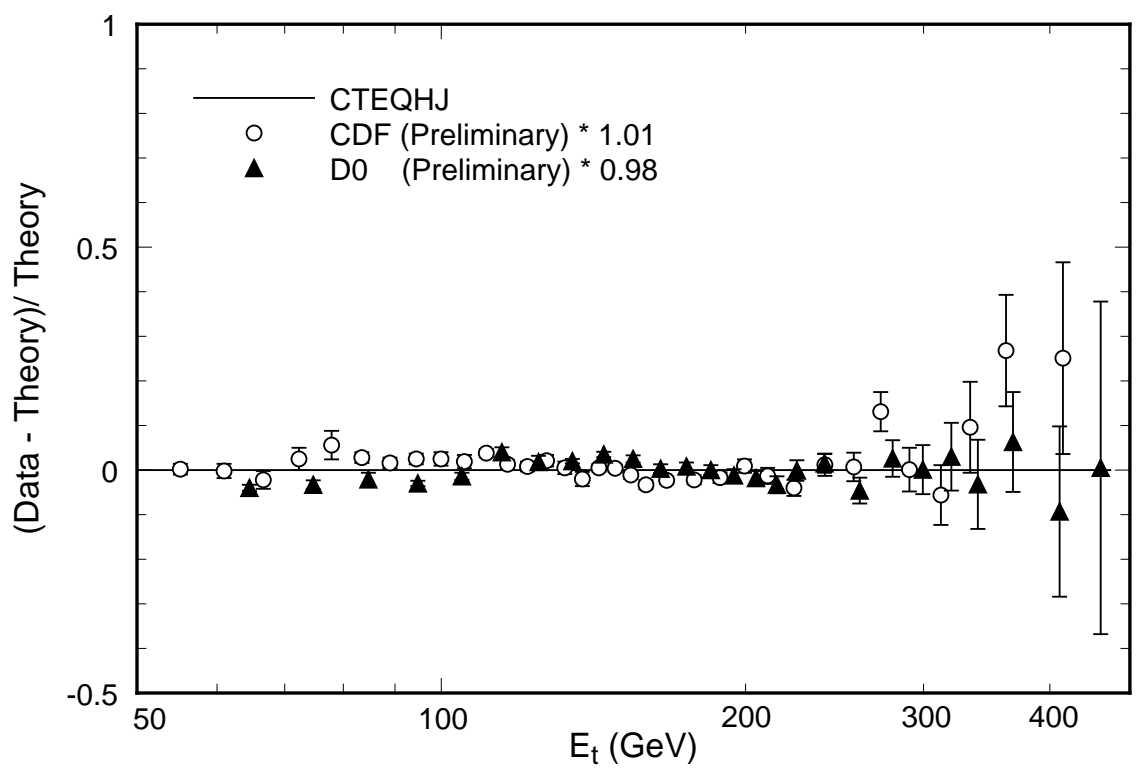

Figure 19 : Inclusive jet cross-section of CDF and D0 compared to NLO QCD calculations based on the CTEQ4HJ parton distributions.

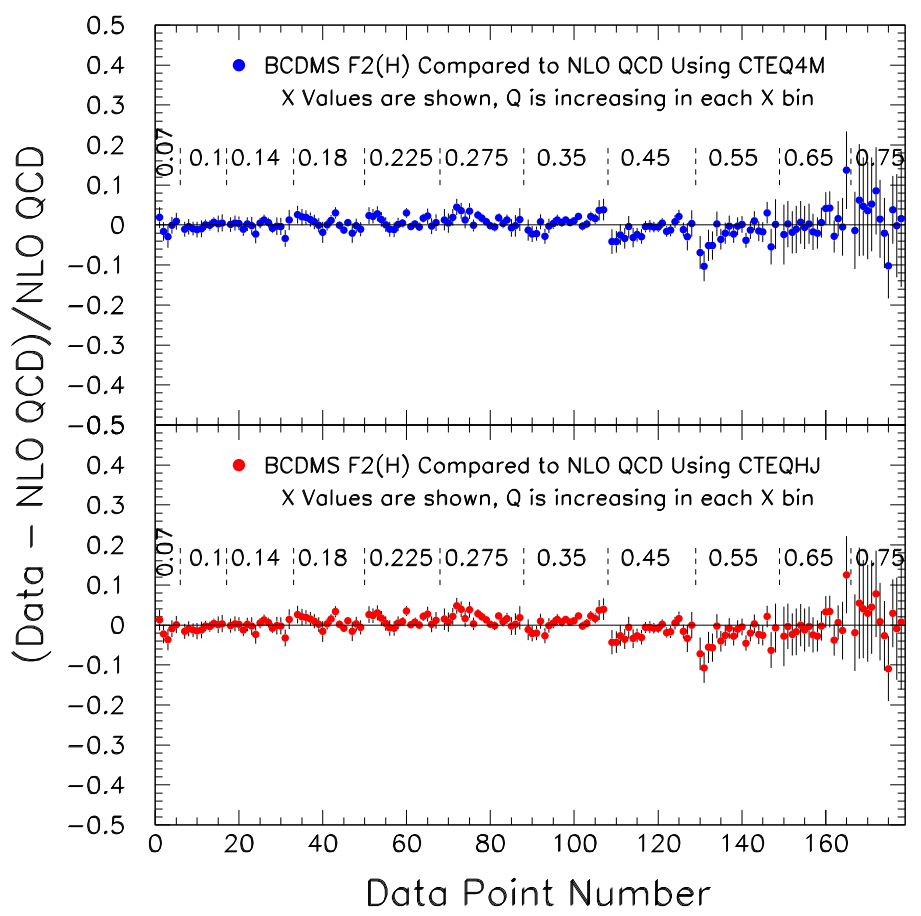

Figure 20 : Percentage deviation of BCDMS proton data from NLO QCD values based on CTEQ4M and CTEQ4HJ. Both PDF sets give good fits. 


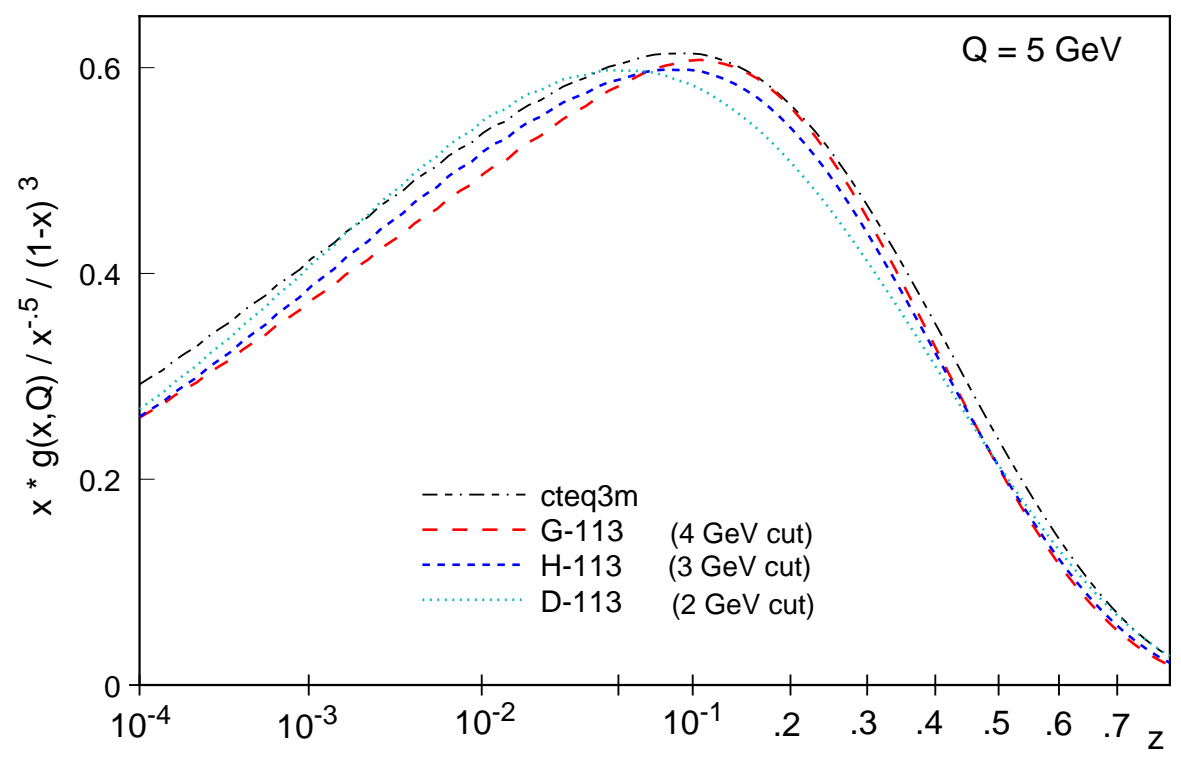

Figure 21 : Comparison of gluon distributions obtained in three global fits using three different values of $Q_{\text {cut }}$ in data selection.

\begin{tabular}{|c|c|c|c|c|}
\hline Series & $\begin{array}{c}\text { New } \\
\text { DIS data }\end{array}$ & $\begin{array}{c}\text { Inclusive } \\
\text { Jet Data }\end{array}$ & $\begin{array}{c}\text { parame- } \\
\text { trization }\end{array}$ & $\begin{array}{c}\text { Section } \\
\text { discussed }\end{array}$ \\
\hline \hline $\mathrm{A}$ & & & $\mathrm{m}$ & 3 \\
\hline $\mathrm{B}$ & $\mathrm{x}$ & & $\mathrm{m}$ & 3,1 \\
\hline $\mathrm{C}$ & $\mathrm{x}$ & & $\mathrm{m}+2$ & 3 \\
\hline CTEQ4A & $\mathrm{x}$ & $\mathrm{x}$ & $\mathrm{m}+2$ & 5 \\
\hline $\mathrm{Q}_{\text {cut }}$ & $\mathrm{x}$ & $\mathrm{x}$ & $\mathrm{m}$ & Appendix \\
\hline
\end{tabular}

Table 1: Several series of global fits on which the physics discussions are based. "New DIS data" refers to those becoming available since 1995. Minimal parametrization "m" refers to Eq. 2; and " $\mathrm{m}+2$ " refers to Eq. 3. The last column refers to the section number where the specific series is discussed. 


\begin{tabular}{|c|c|c|c|c|}
\hline Process & Experiment & Measurable & Data Points & Ref. \\
\hline \multirow[t]{6}{*}{ DIS } & $\overline{\mathrm{BCDMS}}$ & $\overline{F_{2 H}^{\mu}, F_{2 D}^{\mu}}$ & 324 & [26] \\
\hline & NMC & $F_{2 H}^{\mu}, F_{2 D}^{\mu}, F_{2 n / p}^{\mu}$ & 297 & 18 \\
\hline & E665 & $F_{2 H}^{\mu}, F_{2 D}^{\mu}$ & 70 & 21 \\
\hline & H1 & $F_{2 H}^{e}$ & 172 & 19 \\
\hline & ZEUS & $F_{2 H}^{e}$ & 179 & 20 \\
\hline & CCFR & $F_{2 F e}^{\nu}, x F_{3 F e}^{\nu}$ & 126 & 27] \\
\hline \multirow[t]{2}{*}{ Drell-Yan } & E605 & $s d \sigma / d \sqrt{\tau} d y$ & 119 & 28 \\
\hline & NA-51 & $A_{D Y}$ & 1 & 29 \\
\hline W-prod. & $\mathrm{CDF}$ & Lepton asym. & 9 & 30 \\
\hline \multirow[t]{2}{*}{ Direct $\gamma$} & WA70 & $E d^{3} \sigma / d^{3} p$ & 8 & [3] \\
\hline & UA6 & $E d^{3} \sigma / d^{3} p$ & 16 & 31 \\
\hline \multirow[t]{2}{*}{ Incl. Jet } & $\mathrm{CDF}$ & $\overline{c d \sigma / d E_{t}}$ & 36 & [7] \\
\hline & D0 & $d \sigma / d E_{t}$ & 26 & 8] \\
\hline
\end{tabular}

Table 2: List of processes and experiments used in the Global analysis.

\begin{tabular}{|c|c|c|c|}
\hline PDF set & Description & $\alpha_{s}\left(m_{z}\right)$ & $Q_{0}^{2}\left(\mathrm{GeV}^{2}\right)$ \\
\hline \hline & Standard Sets & & \\
\hline CTEQ4M & $\overline{M S}$ scheme & 0.116 & 2.56 \\
\hline CTEQ4D & DIS scheme & 0.116 & 2.56 \\
\hline CTEQ4L & Leading Order & 0.132 & 2.56 \\
\hline \hline & $\alpha_{s}$ series & & \\
\hline CTEQ4A1 & 1 & 0.110 & 2.56 \\
\hline CTEQ4A2 & 2 & 0.113 & 2.56 \\
\hline CTEQ4A3 & Same as CTEQ4M & 0.116 & 2.56 \\
\hline CTEQ4A4 & 4 & 0.119 & 2.56 \\
\hline CTEQ4A5 & 5 & 0.122 & 2.56 \\
\hline \hline & Specials & & \\
\hline CTEQ4HJ & "Hi-Jet" & 0.116 & 2.56 \\
\hline CTEQ4LQ & "Low $\mathrm{Q}_{0} "$ & 0.114 & 0.49 \\
\hline
\end{tabular}

Table 3: List of new CTEQ4 parton distributions and their characteristics. 


\begin{tabular}{|c|c||c|c|c|c|}
\hline Expt. & \#pts & CTEQ4M & CTEQ4HJ & CTEQ4LQ & MRSJ \\
\hline \hline$B C D M S^{H}$ & 168 & $144.8(0.86)$ & $173.0(1.03)$ & $139.4(0.83)$ & $183.1(1.09)$ \\
\hline$B C D M S^{D}$ & 156 & $185.6(1.19)$ & $205.9(1.32)$ & $182.5(1.17)$ & $229.3(1.47)$ \\
\hline$N M C^{H}$ & 104 & $97.3(0.94)$ & $91.7(0.88)$ & $96.0(0.92)$ & $113.4(1.09)$ \\
\hline$N M C^{D}$ & 104 & $93.3(0.90)$ & $90.2(0.87)$ & $97.9(0.94)$ & $122.7(1.18)$ \\
\hline$N M C_{R}$ & 89 & $130.8(1.47)$ & $133.5(1.50)$ & $132.6(1.49)$ & $142.4(1.60)$ \\
\hline$E 665^{H}$ & 35 & $41.3(1.18)$ & $38.5(1.10)$ & $44.5(1.27)$ & $37.8(1.08)$ \\
\hline$E 665^{D}$ & 35 & $32.3(0.92)$ & $33.5(0.96)$ & $34.3(0.98)$ & $29.8(0.85)$ \\
\hline$C C F R F_{2}$ & 63 & $83.2(1.32)$ & $72.4(1.15)$ & $74.3(1.18)$ & $107.7(1.71)$ \\
\hline$C C F R F_{3}$ & 63 & $46.5(0.74)$ & $45.5(0.72)$ & $49.9(0.79)$ & $57.8(0.92)$ \\
\hline$Z E U S$ & 179 & $243.4(1.36)$ & $232.7(1.30)$ & $268.5(1.50)$ & $252.4(1.41)$ \\
\hline$H 1$ & 172 & $118.9(0.69)$ & $120.2(0.70)$ & $131.9(0.77)$ & $109.6(0.64)$ \\
\hline$C D F A_{W}$ & 9 & $4.3(0.48)$ & $3.4(0.38)$ & $3.8(0.42)$ & $3.3(0.37)$ \\
\hline$N A 51$ & 1 & $0.6(0.63)$ & $0.5(0.49)$ & $0.4(0.41)$ & $2.5(2.47)$ \\
\hline$E 605$ & 119 & $97.7(0.82)$ & $101.6(0.85)$ & $100.4(0.84)$ & $97.8(0.82)$ \\
\hline \hline Total & 1297 & 1320 & 1343 & 1356 & 1490 \\
\hline
\end{tabular}

Table 4: Total $\chi^{2}$ values and their distribution among the DIS and DY experiments for current generation of parton distributions which take into account the most recent HERA (1996) and NMC (1995) data. In parentheses are the $\chi^{2} /$ point values. 


\begin{tabular}{|c|c||c|c|c|c|}
\hline Expt. & \#pts & MRSA $^{\prime}$ & CTEQ3M & MRSA & GRV \\
\hline \hline BCDMS $^{H}$ & 168 & $156.9(0.93)$ & $128.7(0.77)$ & $168.0(1.00)$ & $250.3(1.49)$ \\
\hline BCDMS $^{D}$ & 156 & $213.7(1.37)$ & $190.3(1.22)$ & $215.3(1.38)$ & $187.2(1.20)$ \\
\hline NMC $^{H}$ & 104 & $129.0(1.24)$ & $146.6(1.41)$ & $114.4(1.10)$ & $123.8(1.19)$ \\
\hline NMC $^{D}$ & 104 & $151.8(1.46)$ & $137.3(1.32)$ & $135.2(1.30)$ & $115.4(1.11)$ \\
\hline NMC $_{R}$ & 89 & $143.3(1.61)$ & $134.4(1.51)$ & $140.6(1.58)$ & $129.0(1.45)$ \\
\hline E665 $^{H}$ & 35 & $38.2(1.09)$ & $47.6(1.36)$ & $37.8(1.08)$ & $39.9(1.14)$ \\
\hline E665 $^{D}$ & 35 & $29.1(0.83)$ & $44.5(1.27)$ & $29.5(0.84)$ & $29.8(0.85)$ \\
\hline CCFR $F_{2}$ & 63 & $68.0(1.08)$ & $66.2(1.05)$ & $68.7(1.09)$ & $164.4(2.61)$ \\
\hline CCFR $F_{3}$ & 63 & $54.1(0.86)$ & $41.9(0.67)$ & $61.7(0.98)$ & $114.7(1.82)$ \\
\hline ZEUS & 179 & $368.7(2.06)$ & $549.5(3.07)$ & $1222.6(6.83)$ & $843.1(4.71)$ \\
\hline H1 & 172 & $149.5(0.87)$ & $220.2(1.28)$ & $407.6(2.37)$ & $404.2(2.35)$ \\
\hline CDF $A_{W}$ & 9 & $4.2(0.47)$ & $3.0(0.33)$ & $3.7(0.41)$ & $9.6(1.07)$ \\
\hline NA51 & 1 & $0.1(0.06)$ & $0.4(0.42)$ & $0.01(0.01)$ & $0.01(0.01)$ \\
\hline E605 & 119 & $93.5(0.79)$ & $92.6(0.78)$ & $95.9(0.81)$ & $90.3(0.76)$ \\
\hline Total & 1297 & 1600 & 1803 & 2701 & 2502 \\
\hline
\end{tabular}

Table 5: Total $\chi^{2}$ values and their distribution among the DIS and DY experiments for the previous generation of parton distributions which includes experimental data available in 1995 (MRSA') or before 1995 (CTEQ3M, MRSA). GRV does not perform a full global fit. Since it is used widely, it is included here for reference. In parantheses are the $\chi^{2} /$ point values.

\begin{tabular}{|c||c|c|c|c|c|c|}
\hline Parton & $A_{0}$ & $A_{1}$ & $A_{2}$ & $A_{3}$ & $A_{4}$ & \% Momentum \\
\hline \hline$x d_{v}$ & 0.640 & 0.501 & 4.247 & 2.690 & 0.333 & 11.2 \\
\hline$x u_{v}$ & 1.344 & 0.501 & 3.689 & 6.402 & 0.873 & 30.6 \\
\hline$x g$ & 1.123 & -0.206 & 4.673 & 4.269 & 1.508 & 41.7 \\
\hline$x(\bar{d}-\bar{u})$ & 0.071 & 0.501 & 8.041 & 0.000 & 30.000 & - \\
\hline$x(\bar{d}+\bar{u})$ & 0.255 & -0.143 & 8.041 & 6.112 & 1.000 & 13.2 \\
\hline$x s$ & 0.064 & -0.143 & 8.041 & 6.112 & 1.000 & 3.3 \\
\hline
\end{tabular}

Table 6: Parameters for the CTEQ4M initial parton distributions at $Q_{0}=1.6 \mathrm{GeV}$. Also, $\alpha_{s}\left(m_{z}\right)=0.116$, corresponding to $\Lambda_{5}=202 \mathrm{MeV}$. 


\begin{tabular}{|c||c|c|c|c|c|c|}
\hline Parton & $A_{0}$ & $A_{1}$ & $A_{2}$ & $A_{3}$ & $A_{4}$ & \% Momentum \\
\hline \hline$x d_{v}$ & 0.724 & 0.490 & 3.839 & 1.688 & 0.338 & 11.3 \\
\hline$x u_{v}$ & 1.528 & 0.490 & 3.554 & 6.448 & 1.162 & 30.4 \\
\hline$x g$ & 2.141 & -0.058 & 7.554 & 36.405 & 2.223 & 43.7 \\
\hline$x(\bar{d}-\bar{u})$ & 0.054 & 0.490 & 7.200 & 0.000 & 30.000 & - \\
\hline$x(\bar{d}+\bar{u})$ & 0.154 & -0.227 & 7.200 & 6.949 & 1.000 & 11.7 \\
\hline$x s$ & 0.038 & -0.227 & 7.200 & 6.949 & 1.000 & 2.9 \\
\hline
\end{tabular}

Table 7: Parameters for the CTEQ4D initial parton distributions at $Q_{0}=1.6 \mathrm{GeV}$. Also, $\alpha_{s}\left(m_{z}\right)=0.116$, corresponding to NLO $\Lambda_{5}=202 \mathrm{MeV}$.

\begin{tabular}{|c||c|c|c|c|c|c|}
\hline Parton & $A_{0}$ & $A_{1}$ & $A_{2}$ & $A_{3}$ & $A_{4}$ & $\%$ Momentum \\
\hline \hline$x d_{v}$ & 0.702 & 0.443 & 4.003 & 2.433 & 0.622 & 10.9 \\
\hline$x u_{v}$ & 1.226 & 0.443 & 3.465 & 7.589 & 1.146 & 30.1 \\
\hline$x g$ & 0.854 & -0.305 & 3.666 & 1.846 & 1.968 & 41.8 \\
\hline$x(\bar{d}-\bar{u})$ & 0.050 & 0.443 & 6.877 & 0.000 & 30.000 & - \\
\hline$x(\bar{d}+\bar{u})$ & 0.201 & -0.200 & 6.877 & 5.644 & 1.000 & 13.8 \\
\hline$x s$ & 0.050 & -0.200 & 6.877 & 5.644 & 1.000 & 3.5 \\
\hline
\end{tabular}

Table 8: Parameters for the CTEQ4L initial parton distributions at $Q_{0}=1.6 \mathrm{GeV}$. Also, $\mathrm{LO} \Lambda_{5}=181 \mathrm{MeV}$

\begin{tabular}{|c||c|c|c|c|c|c|}
\hline Parton & $A_{0}$ & $A_{1}$ & $A_{2}$ & $A_{3}$ & $A_{4}$ & \% Momentum \\
\hline \hline$x d_{v}$ & 0.852 & 0.573 & 4.060 & 4.852 & 0.693 & 14.7 \\
\hline$x u_{v}$ & 1.315 & 0.573 & 3.281 & 10.614 & 1.034 & 40.4 \\
\hline$x g$ & 39.873 & 1.889 & 5.389 & 0.618 & 0.474 & 31.2 \\
\hline$x(\bar{d}-\bar{u})$ & 0.093 & 0.573 & 7.293 & 0.000 & 30.000 & - \\
\hline$x(\bar{d}+\bar{u})$ & 0.578 & 0.143 & 7.293 & 1.858 & 1.000 & 11.7 \\
\hline$x s$ & 0.096 & 0.143 & 7.293 & 1.858 & 1.000 & 1.9 \\
\hline
\end{tabular}

Table 9: Parameters for the CTEQ4LQ initial parton distributions at $Q_{0}=0.7 \mathrm{GeV}$. Also, $\mathrm{NLO} \Lambda_{5}=174 \mathrm{MeV}$ 


\begin{tabular}{|c||c|c|c|c|}
\hline Expt. & CTEQ4M & CTEQ4HJ & CTEQ4LQ & MRSJ \\
\hline \hline$B C D M S$ & 0.988 & 0.983 & 0.993 & 0.978 \\
\hline$N M C$ & 1.016 & 1.015 & 1.022 & 1.018 \\
\hline$N M C_{R}$ & 1.000 & 1.000 & 1.000 & 1.000 \\
\hline$E 665$ & 1.013 & 1.027 & 1.009 & 1.041 \\
\hline$C C F R$ & 0.976 & 0.971 & 0.983 & 0.968 \\
\hline$Z E U S$ & 1.004 & 0.999 & 1.001 & 1.018 \\
\hline$H 1$ & 0.993 & 0.978 & 0.987 & 0.994 \\
\hline$C D F A_{W}$ & 1.000 & 1.000 & 1.000 & 1.000 \\
\hline$N A 51$ & 1.000 & 1.000 & 1.000 & 1.000 \\
\hline$E 605$ & 1.076 & 1.051 & 1.075 & 1.070 \\
\hline
\end{tabular}

Table 10: List of normalization factors for the experiments which minimize the $\chi^{2}$ 's given in the corresponding $\chi^{2}$ table.

\begin{tabular}{|c||c|c|c|c|}
\hline Expt. & MRSA & CTEQ3M & MRSA & GRV \\
\hline \hline$B C D M S$ & 0.977 & 0.988 & 0.977 & 0.957 \\
\hline$N M C$ & 1.019 & 1.005 & 1.018 & 0.988 \\
\hline$N M C_{R}$ & 1.000 & 1.000 & 1.000 & 1.000 \\
\hline$E 665$ & 1.045 & 0.997 & 1.040 & 0.992 \\
\hline$C C F R$ & 0.968 & 0.976 & 0.968 & 0.949 \\
\hline$Z E U S$ & 1.023 & 0.995 & 1.099 & 0.878 \\
\hline$H 1$ & 0.988 & 0.957 & 1.030 & 0.836 \\
\hline$C D F A_{W}$ & 1.000 & 1.000 & 1.000 & 1.000 \\
\hline$N A 51$ & 1.000 & 1.000 & 1.000 & 1.000 \\
\hline$E 605$ & 1.025 & 1.097 & 1.008 & 1.012 \\
\hline
\end{tabular}

Table 11: List of normalization factors for the experiments which minimize the $\chi^{2}$ 's given in the corresponding $\chi^{2}$ table. 